\title{
ArcheoSciences
}

Revue d'archéométrie

38 | 2014

Varia

\section{Les vitraux alto-médiévaux de Stavelot (Belgique)}

Early medieval stained-glass window from Stavelot (Belgium)

Line Van Wersch, Isabelle Biron, Brigitte Neuray, François Mathis, Grégoire Chêne, David Strivay et Christian Sapin

\section{OpenEdition}

Journals

Édition électronique

URL : http://journals.openedition.org/archeosciences/4284

DOI : $10.4000 /$ archeosciences. 4284

ISBN : 978-2-7535-3691-3

ISSN : 2104-3728

Éditeur

Presses universitaires de Rennes

Édition imprimée

Date de publication : 30 novembre 2014

Pagination : 219-234

ISBN : 978-2-7535-3689-0

ISSN : 1960-1360

Référence électronique

Line Van Wersch, Isabelle Biron, Brigitte Neuray, François Mathis, Grégoire Chêne, David Strivay et

Christian Sapin, "Les vitraux alto-médiévaux de Stavelot (Belgique) », ArcheoSciences [En ligne],

38 | 2014, mis en ligne le 30 novembre 2016, consulté le 03 mai 2019. URL : http://

journals.openedition.org/archeosciences/4284 ; DOI : 10.4000/archeosciences.4284 


\title{
Les vitraux alto-médiévaux de Stavelot (Belgique)
}

\author{
Early medieval stained-glass window from Stavelot (Belgium)
}

\author{
Line VAN Wersch ${ }^{\mathrm{a}, \mathrm{b}}$,, Isabelle Biron ${ }^{\mathrm{d}}$, Brigitte NeuraY, \\ François Mathis ${ }^{c}$, Grégoire ChÊNe ${ }^{c}$, David StrivaY ${ }^{c}$ et Christian Sapin ${ }^{b}$
}

\begin{abstract}
Résumé : Cet article a pour sujet les vitraux alto-médiévaux du monastère de Stavelot. Le nombre très important de fragments, la grande surface de verre représentée et leur qualité de conservation font de Stavelot un site exceptionnel pour l'étude des verres anciens, en particulier ceux du haut Moyen Âge qui restent encore peu connus. Outre leur intérêt pour l'histoire du site et sa compréhension, ces artefacts nous offrent la possibilité d'aborder d'une part l'histoire du vitrail et d'autre part l'histoire des techniques verrières.

L'étude archéologique permet de quantifier et de caractériser les fragments (formes, couleurs, techniques de fabrication). Les analyses en PIXEPIGE nous donnent leurs compositions chimiques. En prime de la coexistence de verre potassique et sodique au sein de mêmes contextes, celle-ci met en exergue des groupes de compositions dans ces deux ensembles révélant des recettes de fabrication variées. Elle nous permet aussi d'aborder les procédés de coloration. Enfin, la question de la production de verre sur le site est évoquée.
\end{abstract}

\begin{abstract}
This paper concerns the early medieval window glass from the monastery of Stavelot. The number of fragments, the surface and the quality of conservation are quite exceptional and Stavelot appears as a very important site for the study of ancient glass, especially glass from the early Middle Ages that is still little known. Beside their interest for the history of the site and its comprehension, these glass artifacts give us the possibility to reach the history of stained-glass window and glass fabrication techniques.

The archaeological study allows us to quantify and characterize the fragments (shapes, colors, shaping techniques). The analyses in PIXE-PIGE provide the glass composition. Further the coexistence of soda and potash glass in the same archaeological contexts, it points out compositional groups revealing various recipes. Thanks to it, we can also reach coloration process. Finally, the question of glass production on the site is debated.
\end{abstract}

Mots clés : Archéologie, haut Moyen Âge, PIXE-PIGE, vitraux, verre.

Abstract: Archaeology, early Middle Ages, PIXE-PIGE, stained-glass window, glass.

\section{INTRODUCTION}

\section{Le site de Stavelot}

L'abbaye de Stavelot (Belgique, province de Liège), fondée au milieu du viI ${ }^{\mathrm{e}}$ siècle par saint Remacle, figure parmi les premières installations monastiques d'Austrasie. Elle connaît un développement remarquable, particulièrement entre les $\mathrm{X}^{\mathrm{e}}$ et $\mathrm{XII}^{\mathrm{e}}$ siècles, et ne sera abandonnée qu'à la fin du $\mathrm{XvIII}^{\mathrm{e}}$ siècle, victime de la tempête révolutionnaire. Depuis 1977, le site fait l'objet de recherches archéologiques qui ont été entamées par des Stavelotains passionnés, puis reprises par l'Association pour la Promotion de l'Archéologie de Stavelot et de sa Région, en collaboration avec l'université de Liège. Actuellement, les travaux sont financés par le Service Public de Wallonie qui entreprend la publication des recherches.

${ }^{a}$ Université catholique de Louvain, CRAN, Louvain-la-Neuve, Belgique. (linevanwersch@gmail.com)

b Université de Bourgogne, UMR Artehis 6298, Dijon-Auxerre, France. (sapin.christian@wanadoo.fr)

cUniversité de Liège, IPNAS - Centre européen d’Archéométrie.(francois.mathis@ulg.ac.be) (gregoire.chene@ulg.ac.be)(dstrivay@ulg.ac.be)

d C2RMF, Équipe mixte Chimie ParisTech-C2RMF, crée dans le cadre de la nouvelle structuration de la recherche à Chimie ParisTech (Institut de Recherche de Chimie Paris - UMR8247 - création au 01/01/2014). (isabelle.biron@culture.gouv.fr)

e Service public de Wallonie - DGO4 - Liège 1 - Service de l'Archéologie. (Brigitte.NEURAY@spw.wallonie.be) 
Les vestiges de trois églises abbatiales - mérovingienne ( $\left(\mathrm{VIII}{ }^{\mathrm{e}} \mathrm{s}\right.$.), carolingienne ( $\mathrm{X}^{\mathrm{e}} \mathrm{s}$.) et ottonienne ( $\mathrm{XI}^{\mathrm{e}} \mathrm{s}$. $)$ - et de deux cloîtres - carolingien ( $\mathrm{x}^{\mathrm{e}} \mathrm{s}$. $)$ et ottonien $\left(\mathrm{XI}^{\mathrm{e}} \mathrm{s}\right.$. $)$ - ont été mis au jour. Selon les sources écrites, dès son arrivée, Remacle érige un oratoire dédié à saint Martin. Il y sera enseveli avant d'être transféré en 685 dans l'abbatiale nouvellement construite. Ces premières constructions seront détruites par les Vikings, dont le passage à Stavelot est signalé en 881 . Au milieu du $x^{e}$ siècle, l'abbé Odilon restaure et agrandit l'abbaye, mais ces nouveaux bâtiments n'auront pas une longue histoire puisqu'au début $\mathrm{du} \mathrm{XI}^{\mathrm{e}}$ siècle l'abbé Poppon entreprend la reconstruction complète de l'abbatiale et des conventuels.

Les vitraux présentés ici appartiennent aux premiers siècles d'occupation monastique, entre le milieu du VII ${ }^{\mathrm{e}}$ siècle et le début du $\mathrm{XI}^{\mathrm{e}}$ siècle. Ils se répartissent en trois secteurs principaux. Le premier (fig. 1, secteur 1) et le plus représentatif (65\% des pièces de verre) est situé à proximité d'un caveau vide attribué à la sépulture primitive de saint Remacle. Les vitraux étaient ponctuellement rassemblés au sein d'un niveau riche en charbon de bois qui recouvrait quelques trous de poteaux et un empierrement, correspondant aux vestiges de l'oratoire Saint-Martin dont le plan précis ne peut être restitué. La présence au-dessus de cette couche, de deux niveaux de sol superposés, attribués respectivement aux $\mathrm{X}^{\mathrm{e}}$ et $\mathrm{XI}^{\mathrm{e}}$ siècle, permet de rattacher la couche contenant les vitraux aux travaux de nivellement consécutifs à l'incendie du monastère par les Vikings en 881. Cet événement est par ailleurs largement documenté sur le site par la présence récurrente d'une couche, témoin soit de l'incendie, soit du nivellement de la démolition de bâtiments incendiés. Ces vitraux étaient associés à un grand nombre de fragments de plombs d'assemblage actuellement en cours d'étude par Chantal Fontaine de l'Institut Royal du Patrimoine Artistique. Le premier examen de ceux-ci révèle la présence de nombreux déchets de fabrication : plombs inachevés, plombs défectueux, barbes et copeaux, têtes de coulées, signalant la proximité d'un atelier de fondeur, mais aussi de mise en plombs de verrières vu les très nombreux fragments de vitraux associés. Cette découverte est capitale car elle tend à situer la fabrication de la majorité des fragments de verre de cette couche dans un laps de temps assez proche de la destruction de l'atelier, dans la deuxième moitié du Ix $\mathrm{x}^{\mathrm{e}}$ siècle.

Les autres pièces de verre plat mises au jour constituent des trouvailles plus disséminées sur le site. Néanmoins, un deuxième secteur se dessine au nord-ouest (fig. 1, secteur 2) où les vestiges d'un petit bâtiment quadrangulaire ainsi que l'amorce d'un second édifice au nord-ouest du précédent sont les seules structures antérieures à l'incendie de 881 . La fonction de ces bâtiments n'est pas connue mais la présence de plusieurs fragments de creusets de verrier et de nombreux déchets liés à la fabrication : verres fondus, scories vitrifiées, éventuels fragments de parois de four, également en cours d'étude par Chantal Fontaine, indique certainement l'existence d'un atelier de verrier. La datation de cette activité ainsi que des fragments de vitraux retrouvés dans ce secteur est plus aléatoire que dans le cas précédent : les tessons étaient répartis dans la couche de démolition consécutive aux destructions vikings ainsi que dans les niveaux directement inférieurs. Ils peuvent donc être datés entre le milieu du VII ${ }^{e}$ siècle et la fin du IX $x^{e}$ siècle.

Le dernier secteur ayant révélé la présence de fragments de vitraux (fig. 1, secteur 3) est perturbé par l'implantation de la grande abbatiale ottonienne du Xi ${ }^{\text {e }}$ siècle qui a systématiquement fractionné les structures construites antérieures et fortement bouleversé les niveaux archéologiques. Les verres plats proviennent de couches archéologiques scellées par les remblais préalables à la construction du XI $\mathrm{X}^{\mathrm{e}}$ siècle et peuvent être attribués à une large phase chronologique entre le milieu du viI ${ }^{e}$ siècle et la fin du $x^{e}$ siècle.

\section{Les vitraux du haut Moyen Âge}

Bien que quelques textes démontrent l'existence de vitraux dès le v siècle (Dell'Acaqua, 2003), la genèse de cet art dans le nord-ouest de l'Europe est encore mystérieuse. Comme le souligne D. Foy et S. Fontaine (2008), le verre de vitrage a jusqu'ici été négligé par de nombreux spécialistes car, pouvant être recyclé, les traces qu'il laisse sont rares et les quelques témoins subsistant sont mis au jour dans les contextes de destruction complexes à appréhender. Néanmoins, plusieurs publications récentes attestent l'existence de vitraux depuis des périodes anciennes.

Si la découpe de feuilles de verre est confirmée dès le $\mathrm{III}^{\mathrm{e}}$ siècle, c'est au IV ${ }^{\mathrm{e}}$ siècle qu'apparaîtraient des morceaux de verre assemblés en compositions géométriques (Foy et Fontaine, 2008). En France, les premiers fragments ont été mis au jour à Saint-Denis dans un contexte du Iv siècle (Rodet-Belarbi, Van Ossel, 2003), puis à Marseille au $\mathrm{V}^{\mathrm{e}}$ siècle (Moliner, 2006) et à Tours, dans la seconde moitié du même siècle (Motteau, 1985). Ces ensembles anciens comptent peu de fragments mais d'autres sites ont livré des collections plus importantes. Sans vouloir en faire une liste exhaustive, nous en retiendrons quelques lots ayant fait l'objet d'analyses archéométriques.

Les sites monastiques de Jarrow et Wearmouth, en Angleterre, sont parmi les premiers à avoir livré des vitraux de la fin du VII ${ }^{e}$ siècle (Cramp, 2006). En Italie, le site du monastère de San Vincenzo al Volturno renfermait de nombreux fragments de verre coloré dans les niveaux datés du 


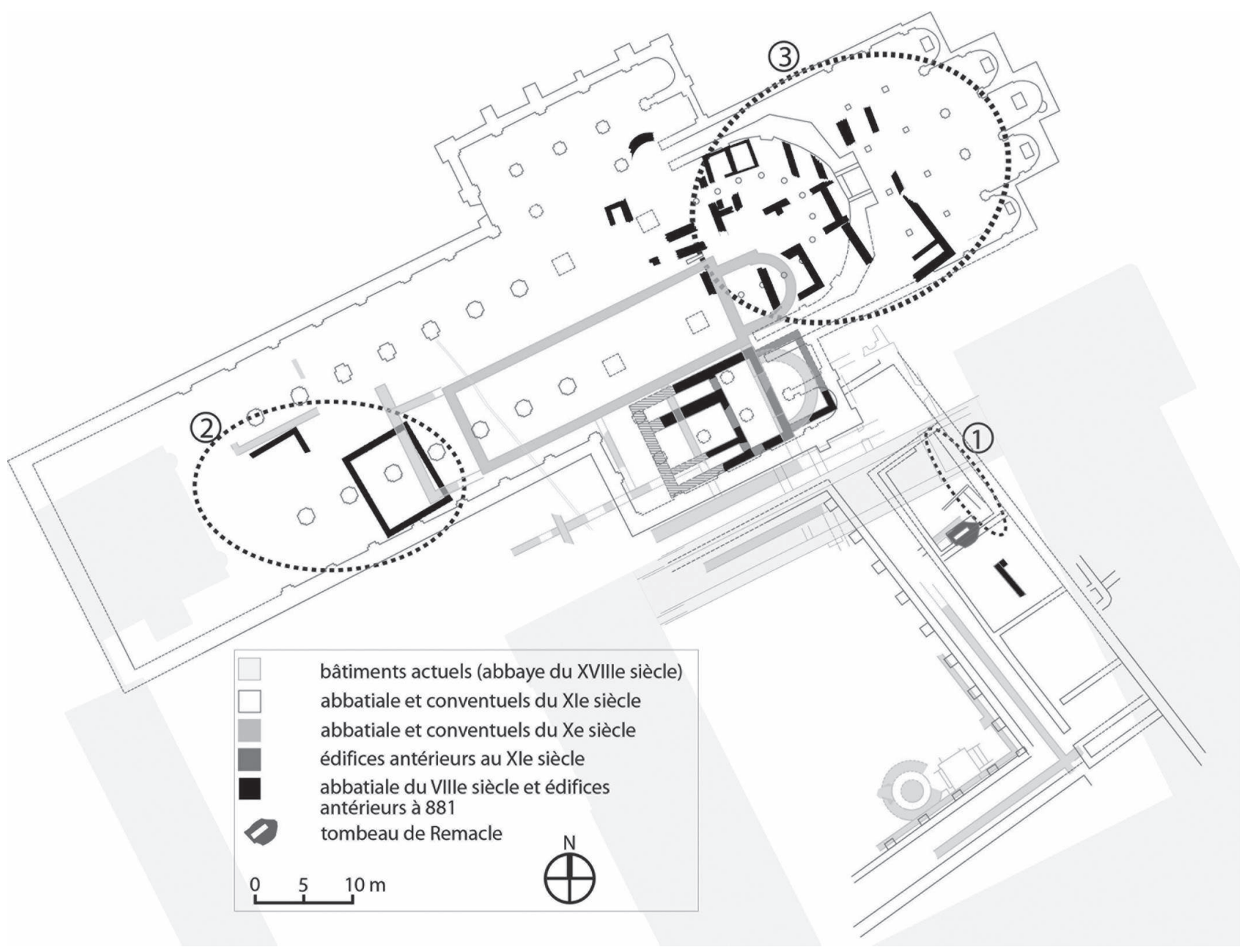

Figure 1 : plan schématique des vestiges des abbatiales et des conventuels de Stavelot et localisation des trois secteurs ayant livré des vitraux antérieurs au XI ${ }^{\mathrm{e}}$ siècle.

Figure 1: schematic plan of Stavelot abbey and conventuals with the three sectors containing window glasses predating the 11th century.

Ix siècle (Dell'Acqua, 1997). Sous l'abbé Joshua (792-817), un atelier y est implanté pour la production de vitraux. Il sera détruit en 881 par des mercenaires sarrasins au service du duc de Naples (Dell'Acqua, 1997). En France, le site épiscopal de Rouen, sans doute construit vers la fin du viII ${ }^{\mathrm{e}}$ siècle et détruit par un raid viking en 841, renfermait aussi des fragments de vitraux (Le Maho, 2001). En Suisse, à Sion, les morceaux de verre coloré sont datés entre le $v^{e}$ et le $\mathrm{VI}^{\mathrm{e}}$ siècle (Wolf et al, 2005). Le site allemand de Paderborn correspond au palais impérial de Charlemagne fondé en 776 et détruit en 778 lors de la première rébellion saxonne. De nombreux fragments de verre et un four de verrier y ont été mis au jour (Gai, 2005). Enfin, à Zalavar, en Hongrie, plusieurs églises bâties au Ix ${ }^{\mathrm{e}}$ siècle et les restes d'un atelier de verriers ont été découverts (Szöke et al., 2004).
Toute cette documentation est d'autant plus importante qu'elle correspond aux prémices d'un bouleversement dans les traditions de fabrication verrières. En effet, depuis le début de notre ère, le verre mis au jour en Europe du nord correspond à celui réalisé à partir d'un mélange de sable et de natron (Henderson, 1985). Ce matériau n'étant pas disponible sur place, les verriers du haut Moyen Âge devaient soit continuer à importer leurs matières premières comme le faisaient leurs prédécesseurs (Foy et al., 2003, Freestone, 2003, Freestone et al., 2000, Freestone et al., 2002, Wedepohl et al., 2003) soit recycler le stock de verre disponible. Si aux $\mathrm{V}^{\mathrm{e}}$ et $\mathrm{VI}^{\mathrm{e}}$ siècles, des importations méditerranéennes atteignent encore le nord-ouest de l'Europe (Freestone et al., 2008), les processus de fabrication et les matériaux sont ensuite moins bien connus jusqu'à la fin du viII siècle 
où apparaissent les premières traces de verre potassique obtenu à partir d'un mélange de sable et de cendres de bois (Wedepohl et al., 2011).

\section{Méthodologie}

La méthodologie appliquée à l'ensemble de Stavelot, repose tout d'abord sur des observations à l'œil nu et sur l'enregistrement des caractéristiques macroscopiques (forme, couleur, état, surface...) dans une base de données informatisée. Celle-ci a ensuite permis le tri et le classement des tessons. Des observations à la loupe binoculaire ont fourni de plus amples informations sur les techniques de façonnage et sur l'état de conservation des artefacts.

Les analyses chimiques ont été effectuées en PIXEPIGE (respectivement émissions de rayons $\mathrm{X}$ et gamma induites par particules) sur la ligne de faisceau extrait ARCHEO de l'Institut de Physique Nucléaire, Atomique et de Spectroscopie (IPNAS) à l'Université de Liège où un cyclotron à énergie variable peut fournir des faisceaux d'ions légers $\left(\mathrm{H}^{+}, \mathrm{D}^{+}, \mathrm{He}^{+}\right)$de 3 à $20 \mathrm{MeV}$. Dans notre cas, un faisceau de protons de $3 \mathrm{MeV}$ est extrait à travers une fenêtre de $\mathrm{Si}^{3} \mathrm{~N}^{4}$ épaisse de $100 \mathrm{~nm}$. Le faisceau de $0,8 \mathrm{~mm}^{2}$ atteint l'échantillon placé à $6 \mathrm{~mm}$ de la fenêtre d'extraction. Dans cet espace, un flux continu d'hélium réduit la dispersion angulaire des particules ainsi que celle en énergie.

Les spectres de rayons $\mathrm{X}$ sont enregistrés par deux détecteurs placés à $45^{\circ}$ par rapport au faisceau incident. Le premier, un détecteur SiLi $\left(30 \mathrm{~mm}^{2}, 132 \mathrm{keV}\right.$ de résolution) équipé d'une fenêtre polymère ultrafine (AP3.7 de chez Moxtek $^{\circledR}$ ), est dédié à la détection des rayons $\mathrm{X}$ de basses énergies émis par les éléments légers ( $\mathrm{Na}, \mathrm{Mg}, \mathrm{Al}, \mathrm{Si}, \mathrm{Ca} . .$.$) .$ L'hélium soufflé entre l'échantillon et la fenêtre du détecteur évite l'absorption des rayons $\mathrm{X}$ de basse énergie par l'air, permettant ainsi de mesurer les éléments jusqu'au sodium $(\mathrm{K} \alpha 1,01 \mathrm{keV})$. Le second détecteur, dédié aux rayons $\mathrm{X}$ de plus forte énergie (6-35 keV), est un détecteur germanium (UltraLeGe de chez Camberra ${ }^{\oplus}, 50 \mathrm{~mm}^{2}, 160 \mathrm{keV}$ de résolution) équipé d'une fenêtre de Béryllium et d'un filtre de $50 \mu \mathrm{m}$ d'aluminium pour absorber les rayons X émis par la matrice. Il est utilisé pour la détection des éléments mineurs et traces (Mathis et al., 2010). Les principales limites de détection ainsi obtenues sont résumées dans le tableau 1.

La PIGE permet de s'affranchir des problèmes de perte de sodium en surface en le détectant en profondeur dans l'échantillon. Pour les rayons gammas, un détecteur germanium coaxial (XtRa HPGe de chez Camberra ${ }^{\circ}$ ) muni d'une fenêtre mince en polymère est placé à $60^{\circ}$ par rapport au faisceau. La détection du sodium est effectuée sur la raie à $440 \mathrm{keV}$ et est quantifiée par rapport au sodium mesuré sur un standard de verre sodique (SRM 620 du National Institute of Standard and Technology - NIST).

Les spectres sont accumulés pendant environ 7 minutes avec un courant de $5 \mathrm{nA}$ pour une dose totale d'environ $2 \mu \mathrm{C}$. Deux spectres minimum sont enregistrés par échantillon, les concentrations d'oxydes majeurs et mineurs $\left(\mathrm{Na}^{2} \mathrm{O}, \mathrm{MgO}, \mathrm{Al}^{2} \mathrm{O}^{3}, \mathrm{SiO}^{2}, \mathrm{P}^{2} \mathrm{O}^{5}, \mathrm{SO}^{3}, \mathrm{~K}^{2} \mathrm{O}, \mathrm{CaO}, \mathrm{TiO} 2\right.$, $\mathrm{MnO}, \mathrm{Fe}^{2} \mathrm{O}^{3}, \mathrm{CoO}, \mathrm{NiO}, \mathrm{Cu}^{2} \mathrm{O}, \mathrm{ZnO}, \mathrm{As}^{2} \mathrm{O}^{5}, \mathrm{Rb}^{2} \mathrm{O}, \mathrm{SrO}$, $\mathrm{ZrO}^{2}, \mathrm{SnO}^{2}, \mathrm{Sb}^{2} \mathrm{O}^{5}, \mathrm{BaO}, \mathrm{PbO}$ ) sont obtenues au moyen du logiciel TRAUPIXE d'AGLAE (Pichon et al. 2010) utilisant le code GUPIXWIN (Campbell et al., 2010) avec une erreur relative de l'ordre de $10 \%$ pour les majeurs et pouvant aller jusqu'à $20 \%$ pour les éléments traces ou mineurs. Les conditions ainsi que les erreurs expérimentales et de calcul sont vérifiées par l'analyse régulière de verres standards (SRM620, 610 et 612 de chez NIST et verre ROUX et MOUSTIER de chez Glaverbel).

Afin de déterminer le corpus à analyser, les échantillons altérés ont été triés et seuls ceux présentant le meilleur état de conservation ont été sélectionnés (fig. 2). Ils ont été fracturés afin d'accéder au verre sain sur la tranche. Pour les fragments choisis, l'épaisseur du verre non-altéré varie entre 2 et $3 \mathrm{~mm}$ environ (fig. 2A). Cette zone est donc de taille supérieure au diamètre du faisceau d'ions. Les morceaux de verre en bon état de conservation ont été analysés sur leur face (fig. 3). Bien qu'aucune altération ne soit visible à l'œil nu, pour éviter toute pollution des résultats, la surface a été abrasée à sec avec une petite meule sur une zone d'environ $1 \mathrm{~cm}^{2}$, puis rincée à l'eau déminéralisée. Dans tous les cas, les échantillons ont été nettoyés à l'éthanol avant analyse. Pour les trente-deux fragments étudiés, nous sommes donc certains que nos mesures concernent le verre sain.

\begin{tabular}{|l|c|c|c|c|c|c|c|c|c|c|c|c|c|c|c|c|c|c|c|c|c|c|c|c|c|c|}
\hline & $\mathrm{Na}$ & $\mathrm{Mg}$ & $\mathrm{Al}$ & $\mathrm{Si}$ & $\mathrm{P}$ & $\mathrm{S}$ & $\mathrm{Cl}$ & $\mathrm{K}$ & $\mathrm{Ca}$ & $\mathrm{Cr}$ & $\mathrm{Mn}$ & $\mathrm{Fe}$ & $\mathrm{Co}$ & $\mathrm{Ni}$ & $\mathrm{Cu}$ & $\mathrm{Zn}$ & $\mathrm{As}$ & $\mathrm{Rb}$ & $\mathrm{Sr}$ & $\mathrm{Y}$ & $\mathrm{Zr}$ & $\mathrm{Nb}$ & $\mathrm{Sn}$ & $\mathrm{Sb}$ & $\mathrm{Ba}$ & $\mathrm{Pb}$ \\
\hline LOD en ppm & 800 & 400 & 368 & 318 & 313 & 297 & 162 & 230 & 400 & 59 & 60 & 65 & 102 & 8 & 10 & 6 & 88 & 26 & 31 & 60 & 82 & 56 & 312 & 307 & 1260 & 30 \\
\hline
\end{tabular}

Tableau 1 : Exemples des limites de détection en ppm des principaux éléments présents dans le verre analysé STV 05.1439.02. Tableau 1: Detection limits in ppm for the elements analyzed in the fragment STV 05.1439.02. 


\section{Résultats}

\section{Observations macroscopiques}

Suite à la fouille et vu leur état d'altération, les fragments de verre plat avaient été conditionnés dans des bocaux contenant de l'eau déminéralisée et du savon Cetavlon ${ }^{\circledR}$ afin d'arrêter le processus de dégradation (Neuray et Lambotte, 2009). Lors d'un premier inventaire, il a été constaté que, malgré ces précautions, plusieurs éléments étaient irrémédiablement perdus. Représentant une dizaine de contenants sur deux cent cinquante, les éléments en verre complètement dilués, réduits en poudre ou recouverts d'algues ne pouvaient plus être manipulés et n'ont pas été retenus pour le comptage.

À Stavelot, trois mille cent trente-six morceaux de verre plat ont été dénombrés. Ils mesurent entre $0,01 \mathrm{~cm}^{2}$ et $46 \mathrm{~cm}^{2}$ et représentent une surface totale d'environ $8845 \mathrm{~cm}^{2}$. Pour comparaison, à Paderborn, plus de deux mille fragments de verre ont été retrouvés sur toute la surface du palais dont une grande majorité en contextes carolingiens (Gai, 2005). Six mille huit cent soixante-quatorze fragments ont été mis au jour sur le site du monastère de San Vincenzo al Volturno dans les niveaux datés du Ix $x^{\mathrm{e}}$ siècle (Dell'Acqua, 1997). À Rouen, deux cents tessons ont été recensés (Le Maho, 2001). Enfin, à Sion, les archéologues mentionnent quatre cent six fragments de verre coloré datés entre le $\mathrm{V}^{\mathrm{e}}$ et le $\mathrm{vI}^{\mathrm{e}}$ siècle (Wolf et al., 2005). Deux cent quatre-vingt-dix-huit pièces ont été comptées à Wearmouth et mille sept cent cinquante-six à Jarrow (Cramp, 2006). Sur ce site, la surface estimée pour les vitres du bâtiment $A$ est de $185 \mathrm{~cm}^{2}$ et de celles du bâtiment D couvriraient environ $2362 \mathrm{~cm}^{2}$ (Cramp, 1975).

Dans le corpus stavelotain, deux grands types de fragments peuvent immédiatement être distingués : ceux opaques altérés (fig. 2) et ceux translucides en bon état de conservation (fig. 3). Les premiers présentent sur chaque face une couche altérée d'environ 0,2 à 4 mm d'épaisseur à l'aspect hétérogène, de couleur blanchâtre à grisâtre. Elle est rendue opaque par l'altération dont la structure en feuillets peut contenir des phases précipitées. Cette altération provient d'une interaction entre le matériau et l'eau de l'environnement de conservation (Biron, 2007). La pénétration de l'eau à la surface du verre conduit à des transformations chimiques et structurales importantes dans la couche hydratée, dont l'épaisseur évolue plus ou moins rapidement selon la composition du verre et les conditions d'enfouissement. D'après les analyses, les verres altérés de notre corpus sont des silicates potassiques - compositions instables chimiquement vis-à-vis de l'hydratation - alors que ceux en bon état sont des silicates sodocalciques - compositions plus stables chimiquement.

\section{Vitraux du groupe 1}
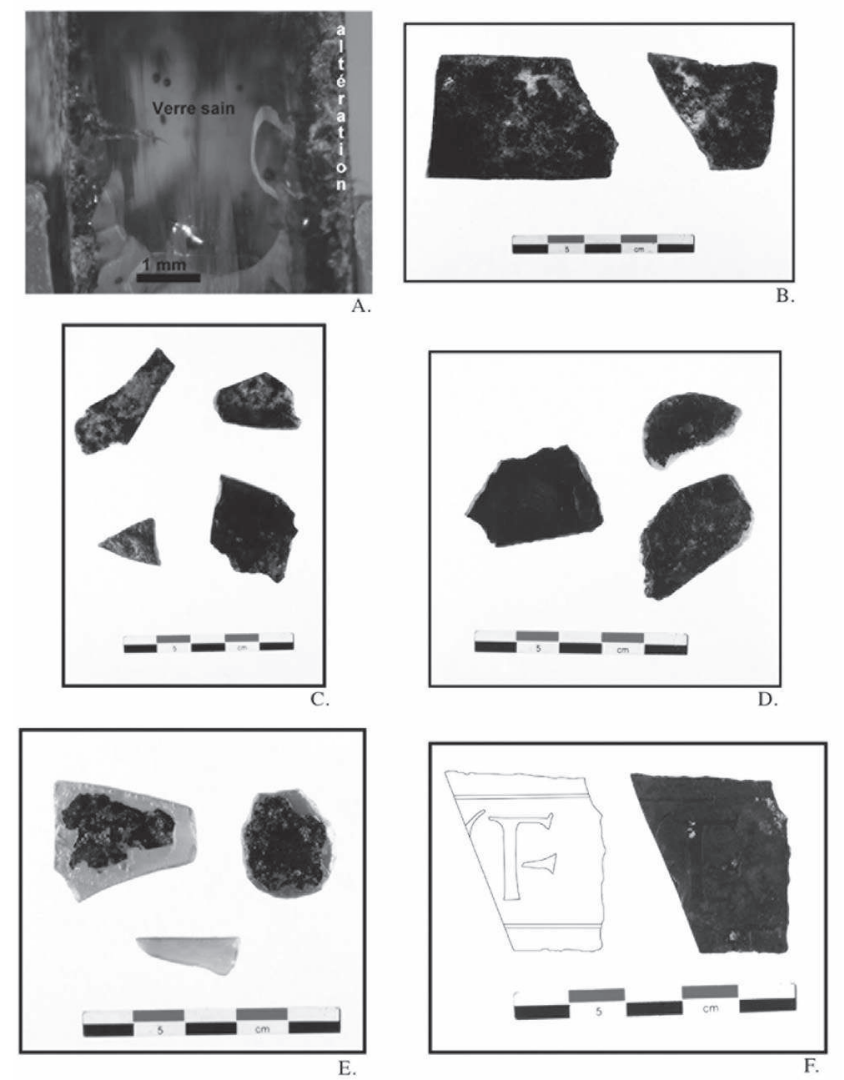

Figure 2: (Voir planche couleur) Vitraux altérés du groupe 1-A : vue au microscope de la coupe d'un tesson avec verre sain et périphérie altérée, $\mathrm{B}$ : verre incolore, $\mathrm{C}$ : verre rose, $\mathrm{D}$ : verre ambre, $\mathrm{E}$ : verre vert, $\mathrm{F}$ : verre avec grisaille.

Figure 2: (See colour plate) Window glass-group 1 A. Microscopic view of a section with healthy glass and altered rims; $B$. Colourless glass; $C$. Pink glass; D. Amber glass; E. Green glass; F. Glass with stain.

Les fragments altérés (fig. 2) forment un premier groupe avec trois mille soixante-six tessons et environ $8645 \mathrm{~cm}^{2}$, soit $96 \%$ de la surface totale et du nombre de fragments. Ils sont particulièrement concentrés dans le secteur 1 (fig. 1). Leur épaisseur $-4 \mathrm{~mm}$ en moyenne - est supérieure à celle des fragments translucides décrits ci-dessous. Pour les échantillons les moins altérés de cet ensemble, environ $10 \% \mathrm{du}$ corpus, la couleur d'origine est encore visible. Il est ainsi possible d'identifier du verre incolore ou verdâtre (couleur naturelle du verre) (fig. 2B), du rouge, du rose (fig. 2C), de l'ambre (fig. 2D) et du vert foncé (fig. 2E). Les tessons verdâtres et verts semblent les plus nombreux.

Les fragments non altérés constituent un second groupe et ne représentent qu'une petite part du mobilier, soit environ soixante-dix fragments et $200 \mathrm{~cm}^{2}(4 \%$ de la surface totale). Ils sont répartis à parts plus ou moins égales entre les 


\begin{tabular}{|c|c|c|c|c|c|c|c|c|c|c|c|c|c|c|c|}
\hline $\mathrm{n}^{\circ}$ d'inventaire & couleur & $\begin{array}{l}\text { état de la } \\
\text { surface }\end{array}$ & type & $\mathrm{Na}_{2} \mathrm{O}$ & $\mathrm{MgO}$ & $\mathrm{Al}_{2} \mathrm{O}_{3}$ & $\mathrm{SiO}_{2}$ & $\mathrm{P}_{2} \mathrm{O}_{5}$ & $\mathrm{SO}_{3}$ & $\mathrm{Cl}$ & $\mathrm{K}_{2} \mathrm{O}$ & $\mathrm{CaO}$ & $\mathrm{TiO}_{2}$ & $\mathrm{MnO}$ & $\mathrm{Fe}_{2} \mathrm{O}_{3}$ \\
\hline STV 99_1278 ambre & ambre & altéré & potassique & 2,91 & 6,43 & 0,92 & 53,67 & 3,17 & 0,13 & 0,42 & 13,56 & 17,52 & 0,08 & 0,45 & 0,34 \\
\hline $\begin{array}{l}\text { STV 99_1278 inco- } \\
\text { lore2 }\end{array}$ & incolore & altéré & potassique & 1,04 & 5,21 & 1,16 & 57,82 & 4,26 & 0,09 & 0,05 & 9,2 & 19,76 & 0,14 & 0,47 & 0,49 \\
\hline $\begin{array}{l}\text { STV 99_1278 inco- } \\
\text { lore3 }\end{array}$ & incolore & altéré & potassique & 1,11 & 5,14 & 1,16 & 56,26 & 4,73 & 0,09 & 0,06 & 9,73 & 20,23 & 0,12 & 0,49 & 0,52 \\
\hline $\begin{array}{l}\text { STV 99_1371 inco- } \\
\text { lore1 }\end{array}$ & incolore & altéré & potassique & 0,74 & 4,5 & 1,84 & 56 & 4,93 & 0,22 & 0,09 & 11 & 18,85 & 0,18 & 0,56 & 0,6 \\
\hline $\begin{array}{l}\text { STV 99_1371 inco- } \\
\text { lore2 }\end{array}$ & incolore & altéré & potassique & 1,05 & 4,82 & 1,56 & 55,11 & 4,31 & 0,18 & 0,1 & 11,82 & 19,32 & 0,15 & 0,64 & 0,61 \\
\hline $\begin{array}{l}\text { STV 99_1278 inco- } \\
\text { lore1 }\end{array}$ & incolore & altéré & potassique & 0,8 & 4,29 & 1,81 & 54,16 & 3,2 & 0,33 & 0,04 & 14,55 & 17,72 & 0,15 & 1,42 & 0,68 \\
\hline STV 99_1371 rose & rose & altéré & potassique & 0,97 & 5,43 & 1,11 & 52,25 & 5,73 & 0,07 & 0,12 & 10,89 & 21,9 & 0,13 & 0,48 & 0,48 \\
\hline STV 99_1371 rouge & rouge & altéré & potassique & 0,71 & 4,08 & 1,15 & 52,23 & 4,33 & 0,25 & 0,18 & 16,94 & 17,83 & 0,11 & 0,34 & 0,8 \\
\hline STV 99_1371 vert1 & vert & altéré & potassique & 0,91 & 3,94 & 1,33 & 51,85 & 4,67 & 0,22 & 0,18 & 16,78 & 16,36 & 0,14 & 0,43 & 0,48 \\
\hline STV 99_1278 vert 1 & vert & altéré & potassique & 0,79 & 4,5 & 1,08 & 53,87 & 4,33 & 0,14 & 0,03 & 10,14 & 21,36 & 0,11 & 0,51 & 0,49 \\
\hline STV99_1371 vert2 & vert & altéré & potassique & 0,74 & 3,99 & 1,12 & 53,02 & 4,59 & 0,2 & 0,27 & 14,42 & 17,98 & 0,14 & 0,58 & 0,44 \\
\hline STV99_1278 vert2 & vert & altéré & potassique & 0,73 & 4,04 & 1,39 & 52,49 & 2,7 & 0,21 & 0,28 & 20,21 & 13,84 & 0,11 & 1,06 & 0,38 \\
\hline STV 93.874 bleu & bleu & bon & sodique & 17,21 & 0,44 & 4,09 & 66,6 & 0,24 & 0,25 & 0,71 & 0,9 & 6,69 & 0,11 & 0,43 & 1,14 \\
\hline STV 99_1278 bleu & bleu & bon & sodique & 15,53 & 0,94 & 2,96 & 64,29 & 0,52 & 0,32 & 0,58 & 2,83 & 8,32 & 0,1 & 0,56 & 1,1 \\
\hline STV 99_1371 bleu & bleu & bon & sodique & 15,14 & 0,59 & 9,25 & 61,29 & 0,25 & 0,19 & 0,44 & 2,31 & 6,85 & 0,08 & 0,56 & 1,19 \\
\hline $\begin{array}{l}\text { STV 99_1371 masse } \\
\text { de verre }\end{array}$ & bleu & bon & sodique & 12,71 & 0,46 & 6,37 & 65,85 & 0,53 & 0,1 & 0,28 & 2,74 & 7,94 & 0,12 & 0,64 & 1,2 \\
\hline STV 94-1260 & turquoise & bon & sodique & 17,07 & 0,6 & 6,31 & 65,18 & 0,09 & 0,09 & 0,38 & 1,11 & 5,96 & 0,13 & 0,48 & 1,07 \\
\hline $\begin{array}{l}\text { STV 05-1557_001 } \\
\text { turquoise }\end{array}$ & turquoise & bon & sodique & 17,44 & 0,62 & 3,04 & 64,52 & 0,18 & 0,3 & 0,64 & 1 & 7,11 & 0,1 & 0,72 & 1,09 \\
\hline STV 90-1867 & turquoise & bon & sodique & 19,95 & 0,19 & 6,35 & 64,27 & 0,07 & 0,18 & 0,59 & 0,76 & 5,36 & 0,09 & 0,27 & 0,77 \\
\hline STV 05-1657-10 & ambre & bon & sodique & 15,99 & 0,61 & 2,76 & 68,61 & 0,11 & 0,11 & 0,82 & 0,79 & 8,87 & 0,25 & 0,22 & 0,93 \\
\hline STV 05-425-035 & incolore & bon & sodique & 14,69 & 0,68 & 3,92 & 67,41 & 0,54 & 0,13 & 0,75 & 1,48 & 8,22 & 0,26 & 0,27 & 1,26 \\
\hline STV 05-602-04 & incolore & bon & sodique & 15,97 & 0,6 & 5,59 & 68,14 & 0,23 & 0,17 & 0,52 & 0,98 & 6,98 & 0,14 & 0,31 & 0,88 \\
\hline $\begin{array}{l}\text { STV 05-1557_001 } \\
\text { incolore }\end{array}$ & incolore & bon & sodique & 16,68 & 0,58 & 2,4 & 67,41 & 0,14 & 0,27 & 0,88 & 0,71 & 9,37 & 0,23 & 0,33 & 0,95 \\
\hline STV 96-9 & incolore & bon & sodique & 15,45 & 0,61 & 8,26 & 64,58 & 0,62 & 0,12 & 0,16 & 1,7 & 7,3 & 0,16 & 0,36 & 1 \\
\hline STV 94-75 & incolore & bon & sodique & 16,26 & 0,86 & 2,65 & 67,76 & 0,16 & 0,2 & 0,66 & 1,49 & 7,74 & 0,16 & 0,49 & 0,94 \\
\hline STV 05-1439-02 & incolore & bon & sodique & 17 & 0,83 & 3,03 & 67,6 & 0,25 & 0,17 & 0,45 & 1,17 & 7,65 & 0,15 & 0,5 & 0,86 \\
\hline STV 05-471-02 & incolore & bon & sodique & 17,09 & 0,58 & 4,16 & 66,49 & 0,51 & 0,26 & 0,81 & 0,7 & 6,07 & 0,11 & 0,55 & 0,94 \\
\hline STV 05-1561 & incolore & bon & sodique & 15,86 & 0,77 & 3,18 & 68,37 & 0,2 & 0,18 & 0,51 & 1,29 & 7,31 & 0,16 & 0,61 & 0,99 \\
\hline STV 1548_16 bleuté & incolore & bon & sodique & 15,2 & 1,02 & 2,35 & 67,67 & 0,22 & 0,19 & 0,66 & 1,28 & 8,34 & 0,18 & 0,66 & 1,07 \\
\hline STV 05-462-01 & incolore & bon & sodique & 16,98 & 0,39 & 10,73 & 60,88 & 1,05 & 0,4 & 0,25 & 1,38 & 6,44 & 0,21 & 0,9 & 1,55 \\
\hline STV 05-606-30 & incolore & bon & sodique & 16,13 & 0,89 & 4,42 & 66,24 & 0,37 & 0,15 & 0,34 & 1,16 & 6,93 & 0,2 & 0,64 & 1,18 \\
\hline $\begin{array}{l}\text { STV 1548_16ver- } \\
\text { dâtre }\end{array}$ & incolore & bon & sodique & 18,03 & 0,88 & 2,45 & 66,73 & 0,09 & 0,27 & 0,65 & 1,12 & 7,62 & 0,14 & 0,84 & 1,17 \\
\hline
\end{tabular}




\begin{tabular}{|c|c|c|c|c|c|c|c|c|c|c|c|c|c|}
\hline $\mathrm{n}^{\circ} \mathrm{d}^{\prime}$ inventaire & $\mathrm{CoO}$ & $\mathrm{NiO}$ & $\mathrm{Cu}_{2} \mathrm{O}$ & $\mathrm{ZnO}$ & $\mathrm{As}_{2} \mathrm{O}_{5}$ & $\mathrm{Rb}_{2} \mathrm{O}$ & $\mathrm{SrO}$ & $\mathrm{ZrO}_{2}$ & $\mathrm{SnO}_{2}$ & $\mathrm{Sb}_{2} \mathrm{O}_{5}$ & $\mathrm{BaO}$ & $\mathrm{PbO}$ & datation du matériel \\
\hline STV 99_1278 ambre & 0 & 0 & 0,03 & 0,04 & 0 & 0,03 & 0,05 & 0,02 & 0,01 & 0,01 & 0,2 & 0 & Antérieur à 881 \\
\hline STV 99_1278 incolore2 & 0 & 0 & 0,1 & 0,04 & 0 & 0,02 & 0,05 & 0,02 & 0,01 & 0 & 0,09 & 0,01 & Antérieur à 881 \\
\hline STV 99_1278 incolore3 & 0 & 0 & 0,16 & 0,04 & 0 & 0,02 & 0,05 & 0,02 & 0,01 & 0 & 0,06 & 0 & Antérieur à 881 \\
\hline STV 99_1371 incolore1 & 0 & 0 & 0,13 & 0,05 & 0 & 0,02 & 0,05 & 0,03 & 0,02 & 0 & 0,15 & 0,02 & Antérieur à 881 \\
\hline STV 99_1371 incolore2 & 0 & 0 & 0,05 & 0,04 & 0 & 0,01 & 0,05 & 0,02 & 0,01 & 0 & 0,16 & 0 & Antérieur à 881 \\
\hline STV 99_1278 incolore1 & 0 & 0,01 & 0,02 & 0,04 & 0 & 0,03 & 0,09 & 0,02 & 0,02 & 0 & 0,6 & 0 & Antérieur à 881 \\
\hline STV 99_1371 rose & 0 & 0 & 0,03 & 0,03 & 0 & 0,03 & 0,05 & 0,02 & 0 & 0,01 & 0,26 & 0 & Antérieur à 881 \\
\hline STV99_1371 rouge & 0 & 0 & 0,5 & 0,19 & 0 & 0,02 & 0,04 & 0,02 & 0,04 & 0,02 & 0,2 & 0,02 & Antérieur à 881 \\
\hline STV99_1371 vert 1 & 0 & 0 & 1,87 & 0,49 & 0 & 0,02 & 0,04 & 0,02 & 0,03 & 0,02 & 0,1 & 0,11 & Antérieur à 881 \\
\hline STV 99_1278 vert 1 & 0 & 0 & 1,89 & 0,31 & 0,01 & 0,01 & 0,04 & 0,02 & 0,25 & 0,02 & 0 & 0,1 & Antérieur à 881 \\
\hline STV 99_1371 vert2 & 0 & 0 & 2,2 & 0,03 & 0 & 0,02 & 0,04 & 0,02 & 0 & 0 & 0,15 & 0,03 & Antérieur à 881 \\
\hline STV 99_1278 vert2 & 0 & 0,01 & 1,36 & 0,44 & 0 & 0,03 & 0,06 & 0,02 & 0,05 & 0 & 0,56 & 0,04 & Antérieur à 881 \\
\hline STV 93.874 bleu & 0,07 & 0 & 0,23 & 0,01 & 0,01 & 0 & 0,07 & 0,02 & 0,09 & 2,08 & 0 & 0,38 & Antérieur au $\mathrm{XI}^{\mathrm{e}}$ siècle \\
\hline STV 99_1278 bleu & 0,06 & 0 & 0,25 & 0,01 & 0 & 0,01 & 0,07 & 0,01 & 0,04 & 1,92 & 0,06 & 0,39 & Antérieur à 881 \\
\hline STV 99_1371 bleu & 0,07 & 0 & 0,25 & 0,02 & 0 & 0,09 & 0,07 & 0,01 & 0,03 & 1,95 & 0,15 & 0,51 & Antérieur à 881 \\
\hline STV 99_1371 masse de verre & 0,06 & 0 & 0,29 & 0,01 & 0 & 0,01 & 0,06 & 0,01 & 0,07 & 1,48 & 0,04 & 0,4 & Antérieur à 881 \\
\hline STV 94-1260 & 0,01 & 0 & 1,7 & 0,05 & 0 & 0 & 0,05 & 0,01 & 0,54 & 0,45 & 0 & 0,79 & Antérieur à 881 \\
\hline STV 05-1557_001 turquoise & 0 & 0 & 1,61 & 0,02 & 0 & 0 & 0,06 & 0,01 & 0,26 & 0,96 & 0,23 & 1,34 & Antérieur à 881 \\
\hline STV 90-1867 & 0,01 & 0 & 1,36 & 0,02 & 0,01 & 0 & 0,05 & 0,01 & 0,18 & 1,23 & 0,05 & 0,99 & Antérieur au $\mathrm{XI}^{\mathrm{e}}$ siècle \\
\hline STV 05-1657-10 & 0 & 0 & 0,03 & 0 & 0 & 0 & 0,02 & 0,03 & 0 & 0,01 & 0,03 & 0,06 & Antérieur à 881 \\
\hline STV 05-425-035 & 0 & 0 & 0,11 & 0,02 & 0 & 0 & 0,03 & 0,02 & 0,08 & 0,06 & 0,03 & 0,23 & Antérieur à 881 \\
\hline STV 05-602-04 & 0 & 0 & 0,06 & 0,01 & 0 & 0 & 0,06 & 0,01 & 0,01 & 0,18 & 0,08 & 0,04 & Antérieur à 881 \\
\hline STV 05-1557_001 incolore & 0 & 0 & 0,03 & 0,01 & 0 & 0 & 0,04 & 0,01 & 0,1 & 0,1 & 0,27 & 0,06 & Antérieur à 881 \\
\hline STV 96-9 & 0,01 & 0 & 0,34 & 0,02 & 0 & 0 & 0,04 & 0,02 & 0,06 & 0,13 & 0,08 & 0,13 & Antérieur à 881 \\
\hline STV 94-75 & 0 & 0 & 0,2 & 0,01 & 0 & 0 & 0,04 & 0,01 & 0,04 & 0,19 & 0,13 & 0,21 & $\begin{array}{l}\text { Antérieur au } \mathrm{XI}^{\mathrm{e}} \text { siècle ou } \\
\text { à } 881\end{array}$ \\
\hline STV 05-1439-02 & 0,01 & 0 & 0,13 & 0,01 & 0 & 0 & 0,05 & 0,01 & 0,05 & 0,16 & 0,11 & 0,2 & Antérieur à 881 \\
\hline STV 05-471-02 & 0,01 & 0 & 0,56 & 0,02 & 0,01 & 0 & 0,06 & 0,02 & 0,13 & 1,27 & 0,08 & 0,91 & Antérieur à 881 \\
\hline STV 05-1561 & 0,01 & 0 & 0,17 & 0,01 & 0 & 0 & 0,04 & 0,01 & 0,06 & 0,19 & 0,06 & 0,31 & Antérieur à 881 \\
\hline STV 1548_16bleuté & 0 & 0 & 0,2 & 0,02 & 0,01 & 0 & 0,05 & 0,01 & 0,18 & 0,23 & 0,21 & 0,48 & Antérieur à 881 \\
\hline STV 05-462-01 & 0,01 & 0 & 0,24 & 0,03 & 0 & 0 & 0,05 & 0,02 & 0,14 & 0,1 & 0,13 & 0,44 & $\begin{array}{l}\text { Antérieur à } 881 \text { ou à } 881 \\
-950\end{array}$ \\
\hline STV 05-606-30 & 0,01 & 0 & 0,12 & 0,01 & 0 & 0 & 0,05 & 0,02 & 0,05 & 0,23 & 0,08 & 0,2 & Antérieur à 881 \\
\hline STV 1548_16verdâtre & 0,01 & 0 & 0,11 & 0,02 & 0,01 & 0 & 0,06 & 0,02 & 0,07 & 0,15 & 0,1 & 0,25 & Antérieur à 881 \\
\hline
\end{tabular}

Tableau 2 : composition des échantillons en \% d'oxydes.

Tableau 2: Glass fragments compositions in \% of oxides.

secteurs 2 et 3 . Ils sont plus fins - en moyenne $2 \mathrm{~mm}$ - et transparents. Légèrement bleutés ou verdâtres, $57 \%$ de l'ensemble peuvent être qualifiés d'incolores (fig. $3 \mathrm{~B}$ et C). Les autres fragments, peu nombreux, sont de couleur turquoise
(4) (fig. 3C), bleu dit " cobalt» (4) (fig. $3 \mathrm{D}$ et E), ou ambre (1 et 4 esquilles) (fig. 3A).

Que ce soit pour les verres dégradés ou pour ceux en bon état, les couleurs observées correspondent à la gamme 


\section{Vitraux du groupe 2}
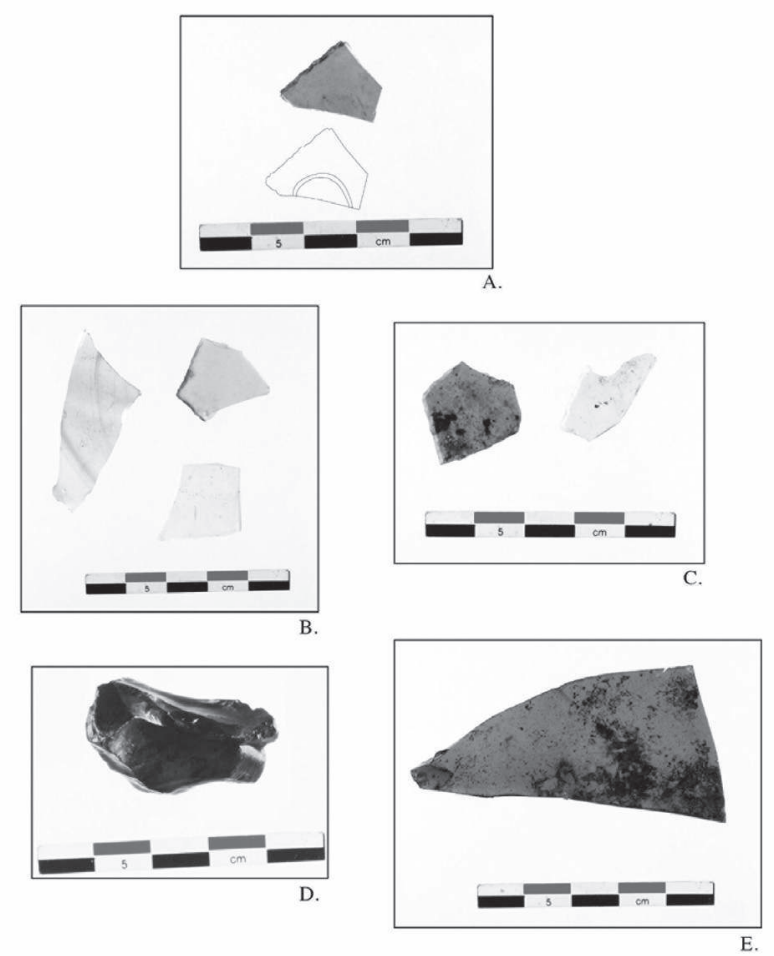

Figure 3 : (Voir planche couleur) Verres non altérés du groupe 2 A : verre ambre avec trace de grisaille; $\mathrm{B}$ : verre incolore bleuté et verdâtre; $\mathrm{C}$ : verre turquoise et incolore bleuté; $\mathrm{D}$ : masse de verre fondu bleu cobalt; $\mathrm{E}$ : verre bleu cobalt.

Figure 3: (See colour plate) Window glass - group 2; A. Amber glass with traces of stain; $B$. Colourless glass, slightly bluish or greenish; $C$. Turquoise and colourless slightly bluish glass; D. Cobalt blue glass chunk; E. Cobalt blue glass.

chromatique connue pour le haut Moyen Âge. Fr. Perrot note que dans la première moitié $\mathrm{du} \mathrm{Ix}^{\mathrm{e}}$ siècle, celle-ci est restreinte à trois ou quatre couleurs et le vert y prédomine (Perrot, 1996). Cette tendance est confirmée par les publications plus récentes (Balcon-Berry et al., 2009; Foy, 2005). À San Vincenzo al Volturno, une grande série de verts et de bleus, de même que du rose pâle et du violet ont été observés, avec en prime des verts et des bleus striés de rouge (Dell'Acqua, 1997). À Zalavar, des fragments sont verts, bleus, brun-mauve, olive (Szöke et al., 2004). À Paderborn, la plupart du verre plat prend une teinte verdâtre (incolore) (Gai, 2005). À Sion, des verres bleus, bleus marbrés de rouge, verts, olive, ambre et incolores sont mentionnés (Wolf et al., 2005).

Lorsqu'elles peuvent être observées, les traces sur les bords et les bulles allongées montrent que les plaques de verre retrouvées à Stavelot ont été obtenues par soufflage.
C'est donc bien la technique du soufflage en cylindre ou au manchon qui a été utilisée, conformément aux pratiques de cette époque décrites plus tard par Théophile. Afin d'obtenir une feuille, l'artisan soufflait un cylindre qu'il coupait ensuite aux deux extrémités puis fendait dans sa longueur pour pouvoir l'ouvrir et le dérouler à plat (Bontemps, 1876). Comme le note D. Foy (2005) pour la plupart des verres plats de cette période, la surface est lisse, souvent brillante sur les deux faces et la matière est beaucoup plus transparente. L'épaisseur des pièces est deux à quatre fois plus mince que celle des verres coulés du haut Empire (Foy, 2005).

$\mathrm{Vu}$ le taux de fragmentation important dû à l'altération, les formes du premier ensemble ne peuvent être restituées. De plus, certains verres sont déformés par la chauffe. On peut cependant distinguer quelques bords grugés témoignant d'une mise en forme. Les calibres sont alors taillés en rectangles, cercles, demi-cercles (fig. $2 \mathrm{E}$ ) et triangles. Plusieurs fragments du second ensemble présentaient également des bords grugés (fig. $3 \mathrm{~A}$ ). Ces éléments sont malheureusement de petite taille et aucune forme précise ne peut être déduite. La technique de découpe des pièces pour être insérées dans des panneaux semble assez ancienne et est décrite par le moine Théophile au début du $\mathrm{XII}^{\mathrm{e}}$ siècle. Selon l'auteur, pour couper le verre, il faut d'abord le faire à chaud, à l'aide d'un fer. Un " grésoir " peut ensuite être utilisé pour corriger les irrégularités (Bontemps, 1876).

Sur plusieurs fragments des deux ensembles, des traces de grisaille ont été repérées. Dans le cas du second groupe, seul un petit fragment ambre portait en négatif une marque laissée par la grisaille (fig. 3A). Dans le premier groupe, des motifs de feuillage ont été observés sur deux fragments et une inscription sur un autre (fig. 2 F). Il se peut que cet ensemble ait contenu plus d'éléments décorés à la grisaille mais leur état actuel empêche la lecture des motifs.

La grisaille est définie comme une sorte d'émail à base d'oxydes métalliques fixés par la cuisson (Perrot, 1996). Au haut Moyen Âge, des traces de grisaille en négatif ont été observées sur plusieurs sites : Paderborn (Gai, 2001), Luxeuil (Pacta, à paraître) et Alézia (Wahlen, 2009). Sur ces petits fragments, le verre est dépoli par endroits et laisse supposer la présence d'une grisaille qui a aujourd'hui disparu. Le cas d'Alézia est particulièrement intéressant puisqu'un visage ainsi qu'un motif végétal peuvent clairement être distingués (Wahlen, 2009). Sur le fragment ambre de Stavelot, aucun motif précis ne peut être reconnu. En revanche, l'inscription offre des comparaisons, notamment à Rouen (Le Maho 2001) et Zalavar (Szöke et al., 2004) où, comme à Stavelot, il s'agit d'une écriture caroline semblable à celle connue dans l'empire carolingien aux VII ${ }^{e}-\mathrm{IX}^{\mathrm{e}}$ siècles. 


\section{Analyses chimiques élémentaires du verre}

Les analyses réalisées sur les trente-deux échantillons issus de contextes datés entre la seconde moitié du viI ${ }^{\mathrm{e}}$ siècle et le début du $\mathrm{IX}^{\mathrm{e}}$ siècle montrent le caractère exceptionnel du site de Stavelot pour l'histoire des techniques verrières (tableau 1).

Les résultats permettent de distinguer deux grands ensembles (fig. 4). Dans le premier, les verres sont des silicates de type potassique et dans le second des silicates de type sodique. Étant donné le lien existant entre la composition chimique et l'état de conservation du verre déjà évoqué précédemment, les fragments altérés du groupe 1 (fig. 2) appartiennent à l'ensemble des compositions potassiques et ceux bien conservés du groupe 2 (fig. 3) ont des compositions sodiques. À Stavelot, nous pouvons donc observer une transition très importante dans l'artisanat verrier du nordouest de l'Europe, celle du changement de fondant, peu connue jusqu'à présent en dehors de l'Allemagne (Wedephol et al., 1997 et Sanke et al., 2002). Sur les autres sites, à Rouen (Le Maho, 2001), Zalavar (Szöke et al., 2004), Sion (Wolf et al., 2005), Jarrow (Cramp, 2006), San Vincenzo (Dell'Acqua, 1997), les fragments alto-médiévaux ont des compositions homogènes et sodiques.

Les analyses en PIXE-PIGE montrent également que ces deux grands ensembles de composition sont hétérogènes (fig. 4 et 5). Diverses recettes ont été employées et témoignent vraisemblablement de phases de fabrication différentes.

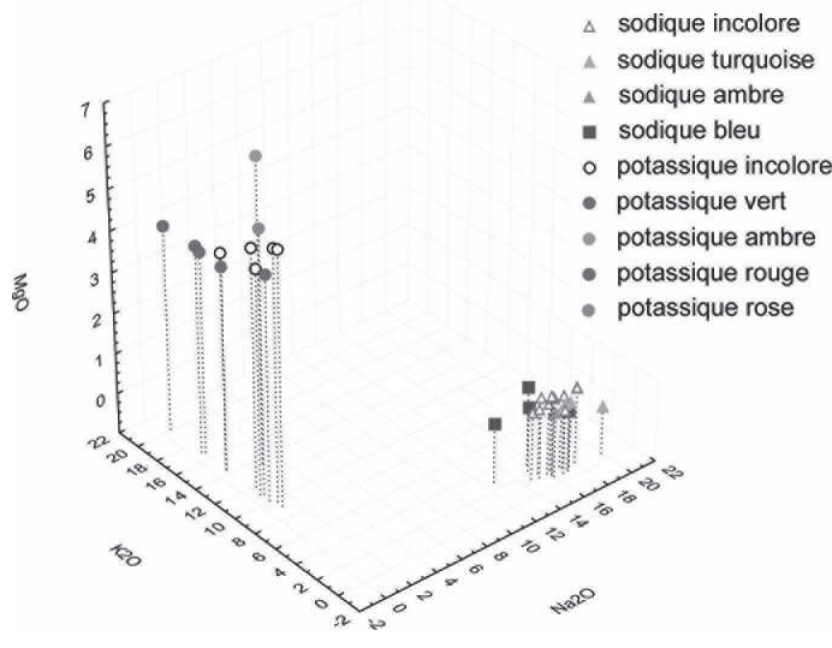

Figure 4 : (Voir planche couleur) Variation des teneurs en oxydes de magnésium, potassium et sodium pour l'ensemble des verres analysés.

Figure 4: (See colour plate) Concentrations of magnesium, potassium and sodium oxides in the analyzed glass fragments.

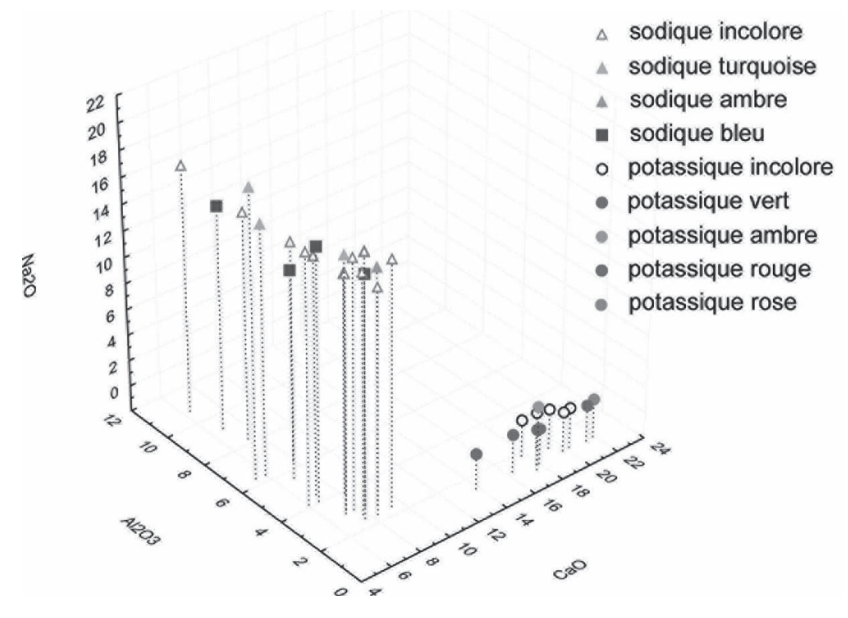

Figure 5 : (Voir planche couleur) Variation des teneurs en oxydes de sodium, aluminium et calcium pour l'ensemble des verres analysés.

Figure 5: (See colour plate) Concentrations of aluminum, calcium and sodium oxides in the analyzed glass fragments.

\section{Fabrication des verres potassiques}

Onze échantillons peuvent être rattachés à cet ensemble. Ces verres sont obtenus à partir d'un mélange de sable et de cendres végétales (Henderson, 1985), en particulier de bois (Wedephol et al., 2011), auquel s'ajoute éventuellement un agent colorant. Une même source de sable a été employée pour tous ces verres, comme en témoignent les corrélations existant entre les teneurs en aluminium (1,08-1,84\%), en titane $(0,11-0,18 \%)$ et en fer $(0,38-0,80 \%)$. En revanche, les sources potassiques se révèlent hétérogènes en proportion et probablement en nature (diverses sortes de bois et/ou modes de préparation), ce que montrent les grandes variations de composition des éléments apportés par ces cendres. Les taux de potassium varient entre 9,20 et 20,21\% (en masse d'oxydes), ceux de magnésium de 3,99 à 6,43\%, ceux de calcium de 21,36 à $13,84 \%$ et ceux de phosphore se situent aux alentours de $4 \%$. Les concentrations de barium $(0,08-0,6 \%)$ et de rubidium (0,014-0,032 \%) sont aussi plus élevées dans ces verres aux cendres de bois que dans ceux utilisant d'autres sources alcalines (Wedephol et al., 2011).

Selon H. Wedephol, la production de verre aux cendres de bois pourrait être subdivisée en trois grandes périodes : 780-1000, 1030-1300 et 1300-1500. Pour la première de ces phases, le taux de potassium est relativement bas, aux alentours de $9,3 \%$ et le taux de sodium avoisine $2,5 \%$. De 1030 à 1300, la concentration de potassium augmente de $10 \%$ et lors de la dernière de ces phases, il diminue à nouveau. À Stavelot, tous les fragments de verre visuellement 
altérés et analysés proviennent du secteur 1 (fig. 1) et peuvent être datés sans ambiguïté avant 881 . Ils se rattacheraient donc à la première phase de production de verre aux cendres de bois. Or, ces fragments se différencient des verres contemporains allemands par deux aspects. Tout d'abord, les verres de Stavelot présentent des concentrations de potassium très variables (de 9 à $20 \%$ ). Dès lors, ces taux ne peuvent plus être uniquement caractéristiques pour une seule période. Ensuite, pour les fragments dont le potassium est le plus bas, le pourcentage de sodium reste inférieur à 2,5\%, ce qui les distingue des verres étudiés par $\mathrm{H}$. Wedephol pour la période 780-1000.

\section{Coloration des verres potassiques}

La composition chimique des verres permet d'identifier les éléments colorants (fig. 6). Les fragments rose et ambre contiennent moins d'oxydes métalliques que les autres couleurs. Le verre ambre est coloré par la combinaison $\mathrm{du}$ fer $(0,34 \%)$ et du soufre $(0,2 \%)$. Le processus de coloration peut-être expliqué par un transfert de charge entre le ligand sulphide $\mathrm{S}^{2-}$ et le cation $\mathrm{Fe}^{3+}$ (Biron et Chopinet, 2013). Le rose est vraisemblablement coloré par de faibles teneurs en oxydes de manganèse $(0,48 \%)$.

Le tesson rouge présente un taux de cuivre plus élevé que celui les éléments incolores, ambre et rose (fig. 6). Pour un fragment $\mathrm{du} \mathrm{XII}{ }^{\mathrm{e}}$ siècle découvert sur le site monastique de Brunshausen, $\mathrm{H}$. Wedephol déduit que du cuivre sous forme métallique a été ajouté au verre cuit en atmosphère réductrice (Wedephol et al., 2011). Lors de la fabrication des verres sodiques, c’est généralement cet élément qui est utilisé pour obtenir un rouge opaque (Nakai et al., 1999; Angelini et al., 2004; Santagostino Barbone et al., 2008; Van Der Werf et al., 2009, Gliozzo et al., 2012). Dans le cas présent, en prime du cuivre, vu leurs taux élevés, le fer, l'étain et l'antimoine pourraient avoir servi d'agents réducteurs pour maintenir le cuivre sous forme métallique. La présence de zinc tendrait à prouver que le cuivre a été introduit dans le verre sous forme de laiton (fig. 6).

La couleur verte résulte également d'une addition de cuivre, supérieure à celle observée dans le fragment rouge (fig. 6). À nouveau, ce type de procédé est bien connu pour le verre sodique (Gliozzo et al., 2012; Heck \& Hoffmann, 2000). Dans cet ensemble contemporain, la recette (nature du cuivre) varie selon les échantillons (fig. 6).

\section{Fabrication des verres sodiques}

Les vingt échantillons rattachés à cet ensemble présentent des compositions hétérogènes. Trois sous-ensembles peuvent être identifiés, d'après la nature des sources alcalines employées (fig. 5).

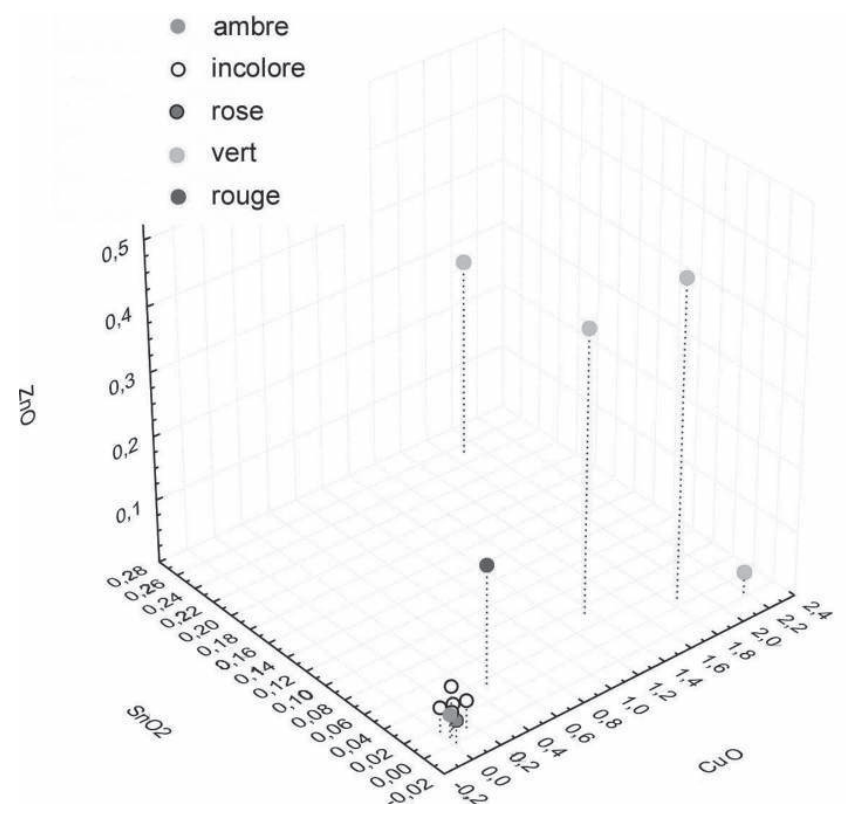

Figure 6: (Voir planche couleur) Variation des teneurs en oxydes de zinc, d'étain et de cuivre pour les verres potassiques analysés.

Figure 6: (See colour plate) Concentrations of zinc, tin and copper oxides in the analyzed glass fragments.

Le premier est constitué de treize fragments (un ambre, un bleu, trois turquoise et huit incolores ou verdâtres). Les recettes de fabrication du verre de base proviennent d'un mélange de sable et de natron (Henderson, 1985). Ces verres sont caractérisés par de faibles teneurs en magnésium $(<1 \%)$, en potassium $(<1,5 \%)$ et en phosphore $(<0,30$ $\%)$. Les variations des teneurs en aluminium $(2,76-6,35 \%)$, en calcium (6,7-9,37\%) et en titane (0,09-0,25\%) pourraient attester de l'usage de diverses sources de sable. Leurs compositions se distinguent de celles des verres romains au natron par des concentrations en calcium sensiblement plus élevées (verres romains $<7 \%$ ), ce qui témoignerait de l'utilisation de sources de sables différentes.

Le deuxième sous-ensemble est formé de trois verres bleus (STV99-1278, STV99-1371 verre plat et STV991371 morceau fondu) et d'un verre incolore (STV96-9). Ils proviennent aussi d'un mélange de sable et de natron mais cette fois des teneurs en potassium (1,70-2,83 \%) et en phosphore $(0,25-0,62 \%)$ sont plus élevées que dans le premier sous-groupe. Celles-ci pourraient résulter de l'introduction, volontaire ou non, de cendres végétales dans la matrice vitreuse. Les teneurs en magnésium restent cependant faibles $(<1 \%)$ et celles en aluminium varient beaucoup plus que dans le groupe précédent (2,96-9,25\%).

Enfin, les 3 derniers verres incolores (STV05-425-035, STV05-462-01, STV05-471-02) sont plus difficiles à clas- 
ser. Leurs compositions different de celles des verres romains au natron par des teneurs en phosphore plus élevées et habituellement observées dans les verres aux cendres de plantes $(0,51-1,05 \%)^{1}$. Pour le fragment STV05-462-01, la concentration en aluminium est aussi très forte $(10,73 \%)$.

\section{Les verres sodiques incolores}

La plupart proviennent du secteur 2 et peuvent être datés avant 881. Trois fragments issus du secteur 3 peuvent être situés avant le $\mathrm{XI}^{\mathrm{e}}$ siècle. Les douze verres qualifiés d'incolores sont verdâtres ou bleuâtres. Ils sont légèrement teintés non pas par une action délibérée de la part des verriers mais par la présence d'impuretés comme le fer dans les sables (fig. 3 $\mathrm{B}$ et $\mathrm{C}$ ).

Pour obtenir ce type de verre après la chute de l'Empire romain, plusieurs scénarios sont actuellement envisagés :

- la continuité des importations : les recherches prouvent qu'au début du haut Moyen Âge (environ 450-550), du verre était encore importé dans le nord-ouest de l'Europe. Des analyses réalisées en Allemagne (Wedepohl et al., 1997), en France (Velde, 1990), en Angleterre (Freestone et al., 2008) et en Belgique (Van Wersch, 2011) montrent pour un large territoire des compositions chimiques assez homogènes. Ces verres pourraient donc être dérivés d'une même matière première correspondant à une seule source de sable provenant sans doute d'une région entre le Nil et le sud d'Israël (Freestone et al., 2008) ;

- le recyclage de verre antérieur : dans l'état actuel de nos connaissances et bien que sujets à certaines discussions, à la fin de l'Antiquité, deux groupes principaux de verres incolores sont attestés dans le nord-ouest de l'Europe (Foster et Jackson, 2009). le groupe dit " HIMT ", caractérisé par sa teneur élevée en titane $(\geq 0,1 \%)$, fer $(\geq 0,7 \%)$ et manganèse ( $\pm 1-2 \%)$, a sans doute été fabriqué au ProcheOrient, voire en Égypte, et diffusé en Europe (Freestone et al., 2005; Leslie et al., 2006; Foy et al., 2003; Aerts et al., 2003; Silvestri et al., 2005; Foster et Jackson, 2009). Le groupe dit "Levantine I " contient moins de sodium $( \pm 15 \%)$ et de fer $( \pm 0,4 \%)$ et plus de calcium $( \pm 9 \%)$ et d'aluminium $( \pm 3 \%)$. Il aurait été produit à partir des sables de la côte syro-palestinienne (Freestone et al., 2000; Foy et al., 2003). En dehors de ces deux grands groupes, le verre "blue-green ", très répandu sur les sites romains, est aussi disponible et aurait pu être recyclé par les verriers du haut Moyen Âge;

1. Le mode de préparation des échantillons pour l'analyse exclut toute contamination superficielle au phosphore.
- de nouvelles productions : on peut aussi supposer qu'un nouveau type de matière première avec des caractéristiques chimiques propres ait été utilisé.

Les verres incolores de Stavelot comparés aux grands groupes de verres connus pour l'Antiquité tardive ne correspondent globalement ni au HIMT ni aux Levantine I. À l'exception de quelques-uns, ils se rapprochent des verres datés entre 450 et 550 ou des verres blue-green. La fabrication des verres incolores de Stavelot pourrait donc représenter une poursuite de la tradition mérovingienne avec l'importation des mêmes matières premières ou bien provenir en partie du recyclage de verres mérovingiens et de verres blue-green. Dans ces derniers, tout comme dans ceux de Stavelot, le manganèse n'est pas ajouté volontairement par les verriers puisque celui-ci doit être présent en quantité supérieure à $0,5 \%$ pour agir comme décolorant (Jackson, 2005).

D'autre part, le recyclage a bien été pratiqué pour une partie des verres de Stavelot car la présence de cobalt et d'antimoine témoigne de l'usage de calcin au natron bleu, de même que la présence de plomb et d'étain. Pour les verres de Stavelot, des mélanges de matières premières conduisent à des compositions marginales, comme le montre également I. Freestone dans son étude sur les verres anglo-saxons (Freestone et al., 2008). Nous retrouvons ainsi diverses associations de sables, de natron et de cendres végétales potassiques, accidentelles ou non.

Outre les diverses sources de sables attestées par les variations des teneurs en aluminium pour l'ensemble des fragments, deux verres (STV05-462-01 et STV96-9) se distinguent par de fortes concentrations : $\mathrm{Al}_{2} \mathrm{O}_{3}=8,26$ et $10,73 \%$. Des verres contenant beaucoup d'aluminium ont été identifiés en Inde, en Asie mineure, notamment à Pergame et Sardis, en Jordanie ainsi qu'en Afrique (Schibille, 2011). À l'heure actuelle, aucun centre de production ne peut être clairement identifié et plusieurs ont dû exister vu la variabilité des recettes et l'étendue de l'aire géographique. Il n'y a sans doute aucun lien entre ceux-ci et les vitraux de Stavelot mais il est intéressant de noter que les exemplaires de Pergame datés aux alentours du viII ${ }^{\mathrm{e}}$ siècle (Schibille, 2011), comme ceux dont il est ici question apparaissent dans une phase de transition pour l'artisanat verrier, entre le verre au natron et celui aux cendres végétales.

\section{Coloration des verres sodiques}

\section{Ambre}

Un seul tesson ambre non altéré est connu sur tout le site de Stavelot. Il porte en négatif les traces d'une grisaille ayant disparu (fig. 3A). Ce fragment de verre sodique ne se distingue des tessons incolores que par un taux de manganèse 
plus bas et de zirconium plus élevé. Sa couleur est produite par la combinaison du fer et du soufre, recette identique au verre ambre potassique.

\section{Turquoise}

Parmi les quatre fragments turquoise mis jour (fig. 3 C), trois d'entre eux ont été analysés (un daté avant 881 et deux avant le $\mathrm{XI}^{\mathrm{e}}$ siècle). Par rapport aux verres « incolores ", les tessons turquoise contiennent plus de cuivre, d'étain, d'antimoine et de plomb. Ces tessons sont colorés au cuivre (1,36-1,70\%), ajouté dans le verre sous forme de bronze. La proportion d'étain est plus importante dans STV94-1260 que dans les deux autres. Bien que proche, la composition de l'alliage varie donc d'un échantillon à l'autre et ce sans rapport avec la chronologie.

À notre connaissance, dans les recettes de coloration turquoise, le cuivre provient toujours de bronze. Il doit être présent à un taux entre 0,5 et $1,5 \%$ pour donner un bleu profond (Brill, 2006). R. Brill (2006) a remarqué que dans de nombreux cas, le matériau colorant dérive d'un alliage de bronze ou de laiton car le cuivre est accompagné d'étain, de zinc et de plomb. À Wearmouth et Jarrow, le cuivre a été introduit dans le verre sous forme d'un alliage binaire de bronze ayant une composition de $89,3 \%$ de cuivre et $10,7 \%$ d'étain ou, plus probablement, sous forme d'un alliage tertiaire de cuivre, étain et plomb (Brill, 2006). En prime des sites anglais, ce type de recette a aussi été repéré dans des contextes du haut Moyen Âge pour des verres turquoise mis au jour à Tongres (Van Wersch et Cosyns, à paraitre) et Luxeuil (Pacta, à paraître).

\section{Bleu}

Ces verres regroupent trois fragments de verre plat (fig. 3E) et une masse de verre informe fondue (fig. 3D). Les tessons plats STV99-1278 et 1371, ainsi que le verre fondu STV99-1371 proviennent des mêmes unités stratigraphiques que les verres potassiques altérés et contrastent par leur très bon état de conservation. Les fragments STV991278 et 1371 sont datés d'avant 881 alors que le verre plat STV93.874 est situé avant le $\mathrm{XI}^{\mathrm{e}}$ siècle.

Il apparait clairement que le cobalt est responsable de la teinte bleue $(0,06-0,07 \%)$. Celui-ci possède un pouvoir colorant élevé, même avec une concentration très faible (Mirti et al., 2002; Brill, 2006). Pour la production de verre bleu, plusieurs sources de cobalt ont été identifiées et caractérisées (Gratuze, 2013). La fabrication des vitraux de cette couleur pourrait également reposer sur le recyclage d'objets antérieurs car, à cette fin, Théophile mentionne la récupération de vaisselle et de tesselles bleues romaines mélangées à du verre incolore (Theophili, 1876). L'existence de ces recettes est prouvée à San Vincenzo (Schibille et Freestone, 2013). C'est également la piste envisagée pour les verres bleus de Chartres, Notre-Dame en Vaux, Mont Saint-Michel, Pavie, York et Glastonbury ( $\mathrm{x}^{\mathrm{e}}-\mathrm{XII}^{\mathrm{e}}$ siècle) dans lesquels Br. Velde voit un mélange de verre de type " antique " et de verre "à cendres de bois " (Velde, 2009).

Parmi les fragments de Stavelot, dans le verre plat STV93874 et le verre fondu STV99-1371, bien que le second contienne plus de potassium que le premier, on observe la même corrélation linéaire entre le cobalt, l'antimoine, le plomb, l'étain et le zinc (par ordre de concentration décroissant). Ils pourraient donc être issus d'une source de cobalt commune impliquant l'exploitation d'un même circuit d'approvisionnement ou le recyclage de mêmes objets. Le type de composition identifiée à Stavelot diffère de l'association cobalt-arsenic-nickel-fer (les teneurs en arsenic et nickel étant de plus très faibles), que l'on retrouve avec l'antimoine, le plomb, l'étain et le zinc dans des sources de cobalt probablement originaires d'Iran et associée aux derniers verres islamiques au natron, datés de la fin du viI ${ }^{\mathrm{e}}$ au début du viII ${ }^{\mathrm{e}}$ siècle (Gratuze, 2013). Cette origine peut donc être écartée. La composition semble aussi différente des sources de cobalt employées pour les tesselles de mosaïques romaines (de type cobalt-fer-nickel-cuivre) (Biron, analyses en cours de publication). Cependant, si le cuivre n'est pas corrélé aux autres traces, il est présent et plusieurs éléments comme l'antimoine pourraient être liés à la réutilisation de tesselles opaques. La composition identifiée à Stavelot ne trouve donc pas vraiment de comparaison mais, à partir de ces deux exemples, les données sont malheureusement insuffisantes pour trancher entre recyclage et nouvelle source de cobalt.

Pour les deux autres fragments bleus (STV99-1371 et 99-1278). Les pourcentages de cobalt, antimoine, plomb et zinc sont très proches, mais la concentration de l'étain est plus faible que dans les deux verres précédents. Aucune corrélation claire n'apparaît entre ces divers éléments. Il semblerait donc que l'origine du cobalt soit différente et, dans le cas du verre 99-1278, le recyclage de verre antique pourrait être envisagé.

Les verres bleus de Stavelot sont donc remarquables à plus d'un titre. En effet, pour des exemplaires chronologiquement proches (antérieurs à 881 et au XI ${ }^{e}$ siècle), trois fragments contiennent une forte proportion de potassium et de phosphore impliquant l'addition de cendres potassiques, volontaire ou non, alors que le dernier tesson a une composition classique de verre au natron. On retrouve donc deux types de fondant alcalin différents mais aussi des sources de cobalt distinctes pour l'obtention du bleu, qu'il s'agisse de recyclage ou non. Les verres bleus ont vraisemblablement été produits avec des matériaux différents et pourraient avoir des 
origines distinctes. Quoi qu'il en soit, ce type de verre bleu réclame de plus amples investigations car, pour le début du Moyen Âge, il pourrait mettre en lumière les procédés de recyclage et/ou de nouvelles importations ainsi que leurs réseaux d'échange.

\section{Production de verre à Stavelot?}

Concernant la réalisation des vitraux, des sources écrites rapportent que lors de la construction de l'abbaye de Wearmouth en 674, des verriers gaulois ont été appelés sur le site par l'abbé pour réaliser les vitraux (Cramp, 2006). Les textes indiquent que les verriers ne restent que peu de temps sur un site; ils font leur travail et puis s'en vont (Cramp, 2006). D'autre part, R. Cramp (2000) mentionne un manuscrit qui relate l'importation de vitres "prêtes à l'emploi " par des évêques de Northumbrie au VIII ${ }^{e}$ siècle à Whithorn. Deux cas de figure semblent donc possibles, la fabrication du verre sur place ou l'importation de verre plat à découper et/ou à assembler.

Sur plusieurs sites du haut Moyen Âge, des ateliers de verriers sont attestés et témoigneraient, de la fabrication de vitres sur place à San Vincenzo al Volturno (Dell'Acqua, 1997), Paderborn (Gai, 2005), Lorsch (Sanke et al., 2002), Zalavar (Szöke et al., 2004). Des restes de fours, des fragments de creusets et des déchets de production constituent les vestiges attestant l'activité des verriers. À San Vincenzo, des tesselles ont été mises au jour dans les niveaux associés à l'atelier, certaines partiellement fondues dans le fond des creusets utilisés pour le verre (Dell'Acqua, 1997). Une tesselle bleue romaine a aussi été trouvée à Zalavar (Szöke et al., 2004). Ceci témoignerait de la réutilisation de tesselles pour la fabrication ou la coloration du verre à vitre.

À Stavelot, des fragments de creusets ont été mis au jour dans les niveaux antérieurs à 881 . Des mosaïques ont aussi été retrouvées mais sans connexion directe avec les creusets. De plus, nous sommes en présence de verre fondu (fig. 3D) et de types de verres différents dont certains ont pu être fabriqués sur le site. Tout laisse donc à penser que des artisans verriers se sont installés à Stavelot. À ce sujet, l'étude des traces de verre sur les creusets ainsi que celle des déchets de fabrication doit être réalisée à l'IRPA et nous apportera certainement des éléments de réponse.

\section{Conclusions}

Stavelot est un site de première importance pour l'histoire de l'artisanat verrier. Le corpus des vitraux alto-médiévaux est important tant du point de vue qualitatif que quantitatif. De plus, il est issu de contextes bien datés entre le milieu $\mathrm{du} \mathrm{VII}^{\mathrm{e}}$ siècle et le début du XI ${ }^{\mathrm{e}}$ siècle. Nous pouvons ainsi approcher une période encore peu connue de l'artisanat verrier et de la production de verre sur un site monastique.

Les observations macroscopiques nous ont permis de distinguer deux grands groupes à partir de l'état de conservation, les éléments très altérés étant dominant tant en nombre qu'en surface. Le verre plat de Stavelot a été obtenu par soufflage au manchon puis découpé à chaud et au grugeoir afin d'obtenir diverses formes. La gamme chromatique est celle connue à l'époque avec une dominance de vert. Sur certaines pièces, des traces de grisaille témoignent de l'existence de cette technique décorative dès le haut Moyen Âge.

Les résultats des analyses chimiques sont primordiaux car ils illustrent, sur le même site, le passage entre le verre sodique et le verre potassique pour un même type d'objet, soit du vitrail. La production verrière de Stavelot apporte donc un éclairage sur la fabrication du verre monumental entre le viI à la fin du IX ${ }^{\mathrm{e}}$ siècle. Les verres de Stavelot témoignent à la fois d'une probable poursuite des traditions verrières mérovingiennes, du recyclage de verres mérovingiens et/ou blue-green romains et de la production de verres d'un nouveau genre. Elle reflète en effet l'émergence de compositions sodiques, avec des mélanges de verres au natron et cendres végétales, fortuites ou non, celles-ci ayant peut-être conduit au verre aux cendres de bois. Elle révèle également la variété des compositions dans ces ensembles, tant sodiques que potassiques, prouvant qu'au haut Moyen Âge, cet artisanat a connu une période de tâtonnement dans la mise au point de nouvelles recettes et ce dans un laps de temps relativement court.

Si le fondant varie, une partie des procédés de coloration semblent subsister. En effet, le cuivre est utilisé comme élément colorant dans les verres sodiques tout comme dans ceux potassiques. L'ambre est également obtenu par des procédés comparables dans les deux types de verre. Le rouge n'est connu que pour le verre potassique. Enfin, les fragments bleus se démarquent du reste du corpus. Ces tessons, seuls éléments sodiques retrouvés dans un ensemble de verres potassiques, sont colorés au cobalt. La provenance de cet élément colorant semble varier mais reste incertaine vu le peu d'exemplaires.

Le lieu de production de tous ces verres ainsi que la présence d'un atelier sur le site doivent encore être discutés et seront certainement éclairés par les autres travaux réalisés sur le reste des artefacts en verre. De même, nous espérons apporter plus de précisions sur le passage entre verre sodique et verre potassique grâce aux études réalisées sur d'autres sites dans le Nord-Ouest de l'Europe, notamment dans le 
Nord de la France ou au Pays-Bas. Enfin, des collaborations accrues avec les historiens pourraient nous permettre de définir la place des artisans ainsi que l'identité et le rôle exact de leurs commanditaires dans la circulation des objets, matériaux et recettes à travers l'Europe du haut Moyen Âge.

\section{Remerciements}

Les auteurs tiennent à remercier la région de Bourgogne ayant financé le post-doctorat dans le cadre duquel cet article a été écrit, l'IPNAS pour le financement des analyses PIXE-PIGE et le Service publique de Wallonie ayant donné sont accord pour le prêt et l'étude des objets.

\section{Bibliographie}

Aerts A., Velde B., Janssens K., Dijkman W., 2003. Change in silica sources in Roman and post-Roman glass. Spectrochimica Acta Part B, 58 : 659-67.

Angelini I., Artioli G., Bellintani P., Diella V., Gemmi M., Polla A., Rossi A., 2004. Chemical analyses of Bronze Age glasses from Frattesina di Rovigo, northern Italy. Journal of Archaeological Science, 31 : 1175-1184.

Biron I., 2007. Le matériau verre et les objets du patrimoine; origine et manifestations des problèmes rencontrés. In Actes du colloque sur la Conservation et restauration du verre. Actualité et problématiques muséales, Trélon, Éd. atelier - musée du verre, 28 septembre 2007, 13-23.

Biron I., Chopinet M. H., 2013. Colouring, decolouring and opacifying of Glass, Chap. 1. 3. In K. Janssens (éd). Modern Methods for Analysing Archaeological and Historical Glass, John Wiley \& Sons, Vol. I, 49-64.

Bontemps G. (trad.), 1876, Thephili presbyteri et monachi, Diversarum Artium Schedula, Liber secundus, Paris.

BriLl R., 2006. Chemical analyses of some glasses from Jarrow and Wearmouth. In R. Cramp (dir.). Wearmouth and Jarrow, Monastics sites, Swindon: English Heritage, 123-147.

Campbell J. L., Boyd N. I. Grassi N., Bonnick P., Maxwell J. A., 2010. The Guelph PIXE software package IV. Nuclear Instruments and Methods in Physics Research Section B: Beam Interactions with Materials and Atoms, 268 (20): 3356-3363.

Cramp R., 2000. Anglo-Saxon window glass. In J. Price (éd.), Glass in Britain and Ireland, Occasional Paper127, British Museum, London, 105-14.

Cramp R., 2006. Window glass. In R. Cramp (dir.). Wearmouth and Jarrow, Monastics sites, Swindon: English Heritage, 56-80.
Dell'Acqua Fr., 1997. Ninth-century window glass from the monastery of San Vincenzo al Volturno (Molise, Italy). Journal of Glass Studies, 39: 33-41.

Foster H.E., Jackson C.M., 2009. The composition of naturally coloured late Roman vessel glass from Britain and the implication for models of glass production and supply. Journal of Archaeological Science, 36: 189-204.

Foy D., 2005. La suprématie du verre soufflé en cylindre : panneaux et vitraux du $\mathrm{v}^{\mathrm{e}}$ au $\mathrm{Ix}^{\mathrm{e}}$ siècle. In $\mathrm{D}$. Foy (éd.), De transparentes spéculations. Vitres de l'Antiquité et du haut Moyen Âge (Occident-Orient), exposition temporaire en liaison avec les $20^{\circ}$ rencontres de l'AFAV sur le thème du verre plat, Bavay, Musée-site d'archéologie, 59-64.

Foy D., Fontaine S., 2008. Diversité et évolution du vitrage de l'Antiquité et du haut Moyen Âge. Un état de la question. Gallia, 65 : 405-459.

Foy D., Picon M., Vichy M., Thirion-Merle V., 2003. Caractérisation des verres de la fin de l'Antiquité en Méditerrannée occidentale : émergence des courants commerciaux. In D. Foy et M.-D. Nenna (éd.), Échange et commerce du verre dans le monde antique. Actes du colloque de l'Association française d'archéologie du verre, Aix-en-Provence - Marseille, 7-9 juin 2001, Montagnac, 41-85.

Freestone I.C., 2003. Primary Glass Sources in the Mid-First Millenium AD. In Annales du $15^{\circ}$ congrès de l'AIHV, New York, 111-115.

Freestone I.C., Gorin-Rosen Y., Hughes M.J., 2000. Primary glass from Israel and the production of glass in Late Antiquity and the early Islamic period. In M.-D. Nenna (dir.), La route du verre : ateliers primaires et secondaires du second millénaire av. J.-C. au Moyen Âge, Lyon, 64-83.

Freestone I.C., Hughes M., Stapelton C.P., 2008. The composition and production of Anglo-Saxon Glass. In V. Evison (dir.), Catalogue of Anglo-Saxon Glass in the British Museum, London, 29-46.

Freestone I.C., Ponting M., Hughes M. J., 2002. The origins of Byzantine glass from Maroni Petrera Cyprus. Archaeometry, 44: 257-272.

Freestone I. C., Wolf S., Thirlwall M., 2005. The production of HIMT glass: elemental and isotopic evidence. In Annales du $16^{e}$ Congrès de l'Association Internationale pour l'Histoire du Verre, London, 2003, 153-157.

GAI S., 2005. Vitres et vitraux du palais impérial de Charlemagne à Paderborn. In D. Foy (éd.). De transparentes spéculations. Vitres de l'Antiquité et du haut Moyen Âge (Occident-Orient), exposition temporaire en liaison avec les $20^{\circ}$ rencontres de l'AFAV sur le thème du verre plat, Bavay, Musée-site d'archéologie, 83-85.

Gliozzo E., Santagostino Barbone A., Turchiano M., Memmi I., Volpe G., 2012. The coloured tesserae decorating the vaults 
of the Faragola balneum (ascoli satriano, Foggia, southern Italy). Archaeometry 54: 311-331.

Gratuze B., 2013. Provenance analysis of Glass Artefact, in Modern methods for analysing Archaeological and Historical glass, chap. 5.1. In K. Janssens (éd). Modern Methods for Analysing Archaeological and Historical Glass, John Wiley \& Sons, 311-343.

Heck M., Hoffmann P., 2000. Coloured opaque glass beads of the Merovingians. Archaeometry, 42: 341-57.

Henderson J., 1985. The raw material of early glass production. Oxford Journal of Archaeology, 4: 267-291.

JACKson C.M., 2005. Making colourless glass in the roman period. Archaeometry 47: 763-780.

Le Maho J., 2001. Les fragments de vitraux carolingiens de Rouen. In Fr. Dell'Acqua, R. Silva (dir.), Il Colore nel Medioevo. Arte simbolo Tecnica. La vetrata in Occidente dal IV all'XI secolo, Actes des journées d'études, Lucques, Villa Bottini, 23-25 sept. 1999, Lucques, Istituto storico Lucchese-Scuola normale superiore di Pisa (coll. Corpus Vitrearum Medii Aevi Italia), 113-124.

Leslie K.A., Freestone I.C., Lowry D., Thirlwall M., 2006. Isotopes in near eastern glass: oxygen by laser fluorination as a compliment to strontium. Archaeometry, 48: 253-270.

Mathis Fr., Othmane G., Vrielynck O., Calvo del Castillo H., Chêne Gr., Dupuis Th., Strivay D., 2010, Combined PIXE/PIGE and IBIL with external beam applied to the analysis of Merovingian glass beads, Nuclear Instruments and Methods in Physics Research Section B: Beam Interactions with Materials and Atoms, 268 (11-12): 2078-2082.

Mirti P., Davit P., Gulmini M., 2002. Colourants and opacifiers in seventh and eighth century glass investigated by spectroscopic techniques. Analytical and Bioanalytical Chemistry, 372: 221-229.

Moliner M., 2006. La basilique funéraire de la rue Malaval à Marseille (Bouches-du-Rhône). In M. Heijmans, J. Guyon (dir.). Dossier : Antiquité tardive, haut Moyen Âge et premiers temps chrétiens en Gaule méridionale, Gallia, 63: 131-136.

Motteau J., 1985. Le verre dans la construction. In Recherches sur Tours, Tours, Association pour le développement des études d'archéologie urbaine de Tours, vol. 4, 39-49.

Nakai I., Numako C., Hosono H., Yamasaki K., 1999. Origin of the red color of Satsuma copper-ruby glass as determined by EXAFS and optical absorption spectroscopy. Journal of the American Ceramic Society, 82: 689-784.

Neuray Br., Lambotte B., 2009. Verre plat : état d'avancement de l'étude des vestiges archéologiques de l'abbaye de Stavelot (Belgique). In S. Balcon-Bérry, F. Perrot, Chr. Sapin (éds). Vitrail, verre et archéologie entre le $\mathrm{v}^{\mathrm{e}}$ et le $\mathrm{XII}^{\mathrm{e}}$ siècle. Actes de la table ronde réunie au Centre d'études médiévales, Auxerre, du 15 au 16 juin 2006, Paris, 153-160.

Pactat I., à paraître, Analyses physico-chimiques de verres plats découverts en Franche-Comté. In D. Billoin (dir.).
L'établissement rural mérovingien de Pratz le Curtillet dans les hautes terres jurassiennes, INRAP-CNRS éditions, Paris.

Perrot Fr., 1996. La couleur et le vitrail. Cahier de civilisation médiévales, 39: 211-215.

Pichon L., Beck L., Walter P., Moignard B., Guillou T., 2010. A new mapping acquisition and processing system for simultaneous PIXE-RBS analysis with external beam. Nuclear Instruments and Methods in Physics Research Section B: Beam Interactions with Materials and Atoms, 268 (11-12): 20282033.

Rodet-Belarbi I., Van Ossel P., 2003. Les épingles à tête anthropomorphe stylisée : un accessoire de la coiffure féminine de l'Antiquité tardive. Gallia, 60: 319-368.

Sanke M., Wedepohl K.H., Kronz A., 2002. Karolingerzeitliches Glas aus dem Kloster Lorsch. Zeitschrift für Archäologie des Mittelalters, 30: 37-75.

Santagostino Barbone A., Gliozzo E., Turchiano M., D’Acapito F., Мemmi Turbanti I., Volpe, G., 2008. Thesectilia panels of Faragola (Ascoli Satriano, southern Italy): a multi-analytical study of the red, orange and yellowglass slabs. Archaeometry, 50: 451-473.

Silvestri A., Molin G., Salviulo G., 2005. Roman and medieval glass from the Italian area: bulk characterization andrelationships with production technologies. Archaeometry, 47: 797-816.

Schibille N., 2011. Late Byzantine mineral soda high alumina glasses from Asia Minor: a new primary glass production group. PLoSONE 6(4): e18970.doi:10.1371/journal. pone.0018970.

Schibille, N., Freestone, I., 2013. Composition, Production and Procurement of Glass at San Vincenzo al Volturno: An Early Medieval Monastic Complex in Southern Italy. PLoS ONE 8(10): e76479. doi:10.1371/journal.pone.0076479.

Sterpenich J., 1998. Altérations des vitraux médiévaux. Contribution à l'étude du comportement à long terme des verres de confinement. Thèse de doctorat, université Henri-Poincaré, Nancy I.

SzöKe B.M., Wedephol K.H., Kronz A., 2004. Silver-Stained Windows at Carolingian Zalavar, Mosaburg (Southwerstern Hungary). Journal of Glass Studies, 46: 85-87.

VAN Wersch L., 2011. Céramique et verre mérovingiens dans la vallée mosane. Apports de l'archéologie et de l'archéométrie à l'histoire économique, sociale et culturelle. Thèse de doctorat, Université de Liège.

Van Wersch L., Cosyns P., à paraître. Het glas. In A. Vanderhoeven (dir.), Opgravingen van de basiliek te Tongeren, Het archeologisch en bouwhistorisch onderzoek in de O.L.V.basiliek van Tongeren (1997-2013). 
Velde Br., 1990. Alumina and calcium oxide contents of glass found in western and northern Europe, first to ninth centuries. Journal of Archaeology, 9: 105-117.

Velde Br., 2009. Composition des vitraux en France du viII ${ }^{\mathrm{e}}$ au $\mathrm{XII}^{\mathrm{e}}$ siècle : de la soude à la potasse. In S. Balcon-Bérry, F. Perrot, Chr. Sapin (éd.) Vitrail, verre et archéologie entre le $v^{e}$ et le XII siècle. Actes de la table ronde réunie au Centre d'études médiévales, Auxerre, du 15 au 16 juin 2006, Paris, 21-26.

Wahlen P., 2009. À propos d'un verre peint trouvé à Alésia (Côted'Or). In S. Balcon-Bérry, F. Perrot, Chr. Sapin (éd.). Vitrail, verre et archéologie entre le $V^{E}$ et le XII siècle. Actes de la table ronde réunie au Centre d'études médiévales, Auxerre, du 15 au 16 juin 2006, Paris, 63-64.

Wedepohl K.H., Gaitzsch W., Follmann-Schulz A. B., 2003. Glassmaking and glassworking in six roman factories in the
Hambach Forest, Germany, Annales du $15^{\circ}$ Congrès de A.I.H.V., New York, 53-55.

Wedepohl K.H., Pirling R., Hartmann G., 1997. Römische und fränkische Gläser aus dem Gräberfeld von Krefeld-Gellep. Bonner Jahrbücher, 197: 177-189.

Wedepohl K.H., Simon KL., Kronz A., 2011. Data on 61 chemical elements for the characterization of three major glass compositions in Late Antiquity and the Middle Ages. Archaeometry, 53: 81-102.

Wolf S., Kesseler C.M., Stern W.B., Gerber Y., 2005. The composition and manufacture or early medieval coloured window glass from Sion (Valois, Switzerland) - a Roman glass making tradition or innovative craftsmanschip? Archaeometry, 47: 361-380. 